Draft Version SEPTEMBer 9, 2021

Preprint typeset using $\mathrm{LATEX}_{\mathrm{E}}$ style AASTeX6 v. 1.0

\title{
A STEREO SURVEY OF MAGNETIC CLOUD CORONAL MASS EJECTIONS OBSERVED AT EARTH IN
} 2008-2012

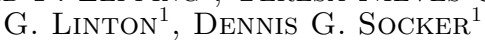

\footnotetext{
${ }^{1}$ Naval Research Laboratory, Space Science Division, Washington, DC 20375, USA; brian.wood@nrl.navy.mil

${ }^{2}$ Heliophysics Science Division, NASA Goddard Space Flight Center, Greenbelt, MD 20771, USA
} \\ ${ }^{3}$ Catholic University of America, Washington, DC 20064, USA
}

Brian E. Wood ${ }^{1}$, Chin-Chun Wu ${ }^{1}$, Ronald P. Lepping ${ }^{2}$, Teresa Nieves-Chinchilla ${ }^{2,3}$, Russell A. Howard ${ }^{1}$, Mark

\begin{abstract}
We identify coronal mass ejections (CMEs) associated with magnetic clouds (MCs) observed near Earth by the Wind spacecraft from 2008 to mid-2012, a time period when the two STEREO spacecraft were well positioned to study Earth-directed CMEs. We find 31 out of 48 Wind MCs during this period can be clearly connected with a CME that is trackable in STEREO imagery all the way from the Sun to near 1 AU. For these events, we perform full 3-D reconstructions of the CME structure and kinematics, assuming a flux rope morphology for the CME shape, considering the full complement of STEREO and $\mathrm{SOHO}$ imaging constraints. We find that the flux rope orientations and sizes inferred from imaging are not well correlated with MC orientations and sizes inferred from the Wind data. However, velocities within the MC region are reproduced reasonably well by the image-based reconstruction. Our kinematic measurements are used to provide simple prescriptions for predicting CME arrival times at Earth, provided for a range of distances from the Sun where CME velocity measurements might be made. Finally, we discuss the differences in the morphology and kinematics of CME flux ropes associated with different surface phenomena (flares, filament eruptions, or no surface activity).

Keywords: Sun: coronal mass ejections (CMEs) — solar wind — interplanetary medium
\end{abstract}

\section{INTRODUCTION}

The term "coronal mass ejection" (CME) was created to describe visible eruptions of mass from the Sun observed in white-light coronagraphic images of the outer solar corona (Tousey 1973; Gosling et al. 1974; Hundhausen et al. 1984; Howard et al. 1985; Kahler 1987) Ever since their discovery, CMEs have been a very active area of solar physics research, due in part to the recognition that CMEs are the cause of most strong, nonrecurrent geomagnetic storms (Gosling 1993). This research has been stimulated by a succession of space-based coronagraphs, particularly the Large Angle Spectrometric COronagraph (LASCO) instrument on board the SOlar and Heliospheric Observatory (SOHO), which has been in continuous operation near Earth's L1 Lagrangian point since 1996 (Brueckner et al. 1995).

However, remote observations are not the only way to study the CME phenomenon. Many spacecraft launched into interplanetary space in the space age have carried plasma and field instruments designed to study the solar wind. Most notable among those specially dedicated to studying the solar wind are Wind (Lepping et al. 1995; Ogilvie et al. 1995) and the Advanced Composition Explorer (ACE) (Stone et al. 1998), launched in 1994 and 1997, respectively, with both spacecraft still currently monitoring the solar wind near L1. The plasma and field instruments on such spacecraft can study solar transients such as CMEs in situ, with in situ detections of CMEs often referred to as "interplanetary coronal mass ejections," or ICMEs.

The CME/ICME distinction represents an implicit acknowledgment that it is not always possible to clearly connect a presumed ICME observed near Earth with a verified CME event observed near the Sun (Lindsay et al. 1999; Russell \& Shinde 2005; Jian et al. 2006). From in situ data alone, there is no definitive procedure for unambiguously distinguishing ICMEs from the background solar wind and other transients such as corotating interaction regions (CIRs) or stream interaction regions (SIRs). Many ICMEs are characterized by a region of low plasma $\beta$ and strong, rotating magnetic fields, and these properties define an ICME subclass called "magnetic clouds" (MCs) (Burlaga et al. 1981; Marubashi 1986; Burlaga 1988; Lepping et al. 1990; Bothmer \& Schwenn 1998).

By far the most favored current interpretation of MCs is that they are magnetic flux ropes (FRs), tube-shaped structures permeated by a helical magnetic field, with legs that stretch back to the Sun (Marubashi 1986; Farrugia et al. 
1995; Bothmer \& Schwenn 1998). Evidence for magnetic connection with the Sun comes from the frequent observations of counterstreaming electron flows within such structures (Gosling et al. 1987; Richardson \& Cane 1996), which is more easily explained with a geometry with field lines bending back towards the photosphere in both directions (as in the FR picture) than with the field line stretching towards the distant reaches of the heliosphere in one direction. In principle, remote sensing should be better suited for studying global CME morphology. Coronagraphic imaging, particularly from SOHO/LASCO, has been used to provide support for the FR paradigm (Chen et al. 1997; Gibson \& Low 1998; Wu et al. 2001; Manchester et al. 2004a; Krall 2007). In particular, large, bright CMEs seen in LASCO images are often found to have large circular rims, which can be interpreted as outlining the apexes of FRs viewed edge-on (Wood et al. 1999; Thernisien et al. 2006).

However, many CMEs present a more confusing appearance in images, making morphological interpretation difficult. By enabling the simultaneous viewing of CMEs from multiple locations, the Solar TErrestrial RElations Observatory (STEREO) mission was designed to improve such analyses. The two STEREO spacecraft, launched in 2006 October, were placed into orbits around the Sun similar to Earth's, but with STEREO-A ahead of Earth in its orbit and STEREO-B behind, and with both spacecraft gradually drifting away from Earth at a rate of about $22^{\circ}$ per year. Each STEREO spacecraft carries four white light telescopes that observe at different distances from the Sun (Howard et al. 2008). There are two coronagraphs, COR 1 and COR2, that observe the Sun's white light corona at angular distances from Sun-center of $0.37^{\circ}-1.07^{\circ}$ and $0.7^{\circ}-4.2^{\circ}$, respectively, corresponding to distances in the plane of the sky of $1.4-4.0 \mathrm{R}_{\odot}$ for $\mathrm{COR} 1$ and $2.5-15.6 \mathrm{R}_{\odot}$ for $\mathrm{COR} 2$. And there are two heliospheric imagers, HI1 and HI2 (Eyles et al. 2009), that monitor the interplanetary medium in between the Sun and Earth, where HI1 observes elongation angles from Sun-center of $3.9^{\circ}-24.1^{\circ}$ and HI2 observes from $19^{\circ}-89^{\circ}$.

Analyses of STEREO data have provided further evidence for a flux rope morphology in CMEs, at least for some events (Thernisien et al. 2009; Möstl et al. 2009; Wood \& Howard 2009). This has led to the suggestion that FRs lie at the core of all CMEs (Vourlidas et al. 2013). Only a fraction of ICMEs are perceived as MCs (Cane \& Richardson 2003), but this might be explained by noting that grazing incidence events may present a messier in situ signature that will not be easily perceived as an MC, and for fast events the shock will subtend a much larger solid angle than the presumed FR driver, meaning that there should be many cases where an ICME shock is observed in situ without ever seeing the driver (Gopalswamy et al. 2009).

The case for the FR paradigm could be greatly strengthened by considering both in situ and imaging constraints for a variety of events and demonstrating conclusively that the FR shapes inferred for these events from imaging are consistent with the MC encounter times and FR orientations inferred in situ. The STEREO spacecraft once again provide an excellent opportunity to do this, especially given their heliospheric imaging capabilities, which allow CMEs to be tracked all the way to $1 \mathrm{AU}$, where they can be observed in situ by ACE, Wind, or by the STEREO spacecraft themselves. A CME initiated on 2008 June 1 that ended up striking STEREO-B on 2008 June 6 provided a perfect opportunity for such an assessment, and for this event the inferences of FR size and orientation from the imaging and in situ data are in very good agreement (Möstl et al. 2009; Wood et al. 2010a). However, it is far from clear that this is the norm. We here present a survey of Earth-directed events that can address this issue more fully.

Our goal is to use STEREO and LASCO data to provide a full 3-D kinematic and morphological analysis of a sample of CMEs observed as MCs at Earth, specifically by Wind. Aside from providing a nice sample of events to study the characteristics of CMEs in the STEREO era, we seek to address two questions in particular: 1 . What percentage of MCs detected at $1 \mathrm{AU}$ can be definitively connected to CMEs observed erupting from the Sun, and 2. When CME images are analyzed in the context of the FR paradigm, are the inferred FR structures consistent with the characteristics of the MCs observed in situ?

\section{CONNECTING MAGNETIC CLOUDS WITH CORONAL MASS EJECTIONS}

For our study, we confine our attention to a time period when the STEREO spacecraft were well located to observe Earth-directed CMEs. We want events well constrained by STEREO and SOHO/LASCO imagery, and we want CMEs trackable out to distances close to $1 \mathrm{AU}$ in the HI2 field of view. The requirement of being able to track CMEs far from the Sun is in part simply due to a desire to study CME kinematics far from the Sun, and in part because tracking CMEs all the way to $\sim 1 \mathrm{AU}$ in images should maximize our ability to connect an MC with its corresponding CME. It is somewhat arbitrary where to draw the line between a good viewing geometry and a poor one, but in general the first year of STEREO operations in 2007 was poor because the STEREO spacecraft were still too close to Earth to stereoscopically study Earth-directed events very well, and the post-2012 time period was generally poor because the STEREO spacecraft had by then drifted too far $\left(>130^{\circ}\right)$ from the Sun-Earth line to effectively study and track Earth-directed CMEs far from the Sun (Lugaz et al. 2012b). 
Table 1. CMEs Associated with Wind MCs

\begin{tabular}{|c|c|c|c|c|c|c|c|c|c|c|c|c|}
\hline \multirow[b]{2}{*}{ ID } & \multirow[b]{2}{*}{ CME Start } & \multirow[b]{2}{*}{ ICME Arrival } & \multirow[b]{2}{*}{$\begin{array}{c}\phi_{l}{ }^{a} \\
(\mathrm{deg})\end{array}$} & \multirow[b]{2}{*}{$\begin{array}{c}\theta_{l}^{a} \\
(\mathrm{deg}) \\
\end{array}$} & \multirow[b]{2}{*}{$\mathrm{Q}_{l}{ }^{a}$} & \multirow[b]{2}{*}{$\begin{array}{c}\phi_{n}{ }^{b} \\
(\operatorname{deg})\end{array}$} & \multirow[b]{2}{*}{$\begin{array}{c}\theta_{n}{ }^{b} \\
(\mathrm{deg})\end{array}$} & \multirow[b]{2}{*}{$\mathrm{Q}_{n}{ }^{b}$} & \multicolumn{4}{|c|}{ Surface Activity } \\
\hline & & & & & & & & & Type $^{c}$ & Time & $\begin{array}{c}\lambda_{p}{ }^{d} \\
(\mathrm{deg})\end{array}$ & $\begin{array}{c}\beta_{p}{ }^{d} \\
(\mathrm{deg}) \\
\end{array}$ \\
\hline 2 & 2009-06-22Т04:15 & 2009-06-27T12:00 & 52 & 42 & 3 & 257 & 65 & 3 & none & & & \\
\hline 3 & 2009-07-15Т04:30 & 2009-07-21T00:00 & 297 & -17 & 2 & 335 & 4 & 3 & none & & & \\
\hline 5 & 2009-09-25T12:00 & 2009-09-30Т02:00 & 78 & 51 & 2 & 110 & 18 & 1 & none & & & \\
\hline 6 & 2009-10-27Т09:00 & 2009-11-01Т04:00 & 352 & 58 & 2 & 42 & 18 & 3 & none & & & \\
\hline 7 & 2009-12-06Т02:30 & 2009-12-12Т06:00 & 233 & -35 & 3 & 217 & -3 & 3 & none & & & \\
\hline 8 & 2010-04-03Т09:10 & 2010-04-05Т09:00 & 173 & 57 & 2 & 219 & -1 & 3 & $\mathrm{FL}(\mathrm{B} 7.4), \mathrm{FE}$ & 9:04 & 2 & -21 \\
\hline 9 & 2010-05-23Т16:35 & 2010-05-28Т03:00 & 125 & -81 & 1 & 78 & -70 & 2 & $\mathrm{FL}(\mathrm{B} 1.3), \mathrm{FE}$ & $16: 52$ & 12 & 19 \\
\hline 13 & 2010-10-26Т02:00 & 2010-10-30T10:00 & 153 & 60 & 3 & 169 & 73 & 1 & $\mathrm{FE}$ & $0: 30$ & 1 & -55 \\
\hline 14 & 2010-12-14Т05:00 & 2010-12-19Т20:00 & 319 & 24 & 3 & 285 & -19 & 3 & none & & & \\
\hline 15 & 2011-02-15Т01:55 & 2011-02-18Т02:00 & 11 & -4 & 3 & 53 & -72 & 3 & $\mathrm{FL}(\mathrm{X} 2.2), \mathrm{FE}$ & $1: 44$ & 12 & -7 \\
\hline 16 & 2011-03-24T19:00 & 2011-03-29T16:00 & 294 & 7 & 2 & 276 & 22 & 2 & none & & & \\
\hline 17 & 2011-05-25Т04:20 & 2011-05-28Т07:00 & 112 & -29 & 1 & 114 & -25 & 2 & FL(B1.9) & $3: 41$ & 12 & -16 \\
\hline 18 & 2011-06-02Т07:45 & 2011-06-04T21:00 & 317 & 20 & 3 & 274 & -17 & 2 & $\mathrm{FL}(\mathrm{C} 3.7), \mathrm{FE}$ & $7: 22$ & -24 & -15 \\
\hline 19 & 2011-06-14Т06:10 & 2011-06-17Т03:00 & 18 & 66 & 3 & 208 & 17 & 3 & FE & 3:00 & -54 & -13 \\
\hline 20 & 2011-09-13Т22:10 & 2011-09-17Т04:00 & 272 & -27 & 3 & 208 & 9 & 2 & FL & $22: 00$ & 3 & 10 \\
\hline 21 & 2011-09-13Т22:10 & 2011-09-17Т04:00 & 335 & -12 & 3 & 184 & 5 & 3 & FL & $22: 00$ & 3 & 10 \\
\hline 28 & 2012-07-04T16:45 & 2012-07-08Т03:00 & 160 & -40 & 2 & 160 & -51 & 1 & FL(M1.8) & $16: 33$ & 33 & 6 \\
\hline
\end{tabular}

Note- ${ }^{a} \mathrm{MC}$ solution from Lepping et al. (2011, 2015), with $\left(\phi_{l}, \theta_{l}\right)$ the MC axis direction in HEE coordinates, and $Q_{l}$ a fit quality flag. ${ }^{b} \mathrm{MC}$ axis direction $\left(\phi_{n}, \theta_{n}\right)$ in HEE coordinates and fit quality flag $\left(Q_{n}\right)$ from an analysis newly presented here. ${ }^{c}$ Type of surface activity: $\mathrm{FL}=$ flare (with GOES designation if available), $\mathrm{FE}=$ filament eruption. ${ }^{d}$ Longitude and latitude of surface activity in HEE coordinates.

On 2012 July 12, STEREO-A reached a longitudinal distance of $120^{\circ}$ from the Earth, which we decide to mark the end of our survey time period, although STEREO-B would not reach this angular distance from Earth for another 3 months. As for the beginning of the survey period, we decided to avoid 2007 but to consider all MC events in 2008. Thus, the first step in defining our sample of events was to identify MCs observed by Wind between 2008 January 1 and 2012 July 12. Our sources are the Wind MC lists from Lepping et al. (2011, 2015), involving the use of both an automated procedure (Lepping et al. 2005) and visual inspection to find MCs in the Wind database. Wu \& Lepping $(2015,2016)$ have studied some statistical properties of these MC lists.

There are a total of $48 \mathrm{MCs}$ identified in the time period of interest. The next step was to peruse the STEREO and SOHO/LASCO imagery to identify CME candidates for these MCs. Our approach was to start far from the Sun in the HI2 field of view, in which Earth is actually visible. For each MC, we look at movies of HI2-A and HI2-B images near the time of the MC to see if we can see a front passing over Earth at about that time, which we then seek to 
track backwards to HI1, then to COR2, and finally to COR1 to identify the CME associated with that front and its initiation time at the Sun. Only if we can identify a CME trackable continuously from COR1 to HI2 that appears to reach Earth at about the right time do we consider ourselves to have a candidate CME for the MC. It is of course simplistic to expect to see a front on top of Earth at the exact time of the MC, as the apparent position of a CME front depends on its projection into the 2-D image plane. In practice, the $\mathrm{MC}$ will be more likely observed some time after the front is observed passing over Earth due to these projection effects, and this time delay can become larger the farther the STEREO spacecraft are from Earth. These projection effects are naturally considered when identifying candidate CMEs.

For candidate CMEs, STEREO and SOHO/LASCO imagery are used to perform a full 3-D kinematic and morphological reconstruction of the CME, which will be described in detail in the next section. Only after such a reconstruction is deemed successful at both adequately reproducing the appearance of the CME in images, and in adequately reproducing the CME's arrival time at Wind, is the MC-CME connection finally deemed secure. In some cases, there are multiple CME candidates for an MC, and multiple CMEs must therefore be modeled in order to establish the one most likely to be responsible for the MC. It is worth noting that there were a few cases where a CME considered a candidate based on the initial inspection of the images was ultimately deemed incorrect once a detailed reconstruction was performed, the point being that it is possible to be fooled into erroneous CME-ICME connections if analysis is limited to visual inspection only.

Nevertheless, most of our candidate CMEs were confirmed, and we ultimately connect 31 out of the 48 Wind MCs with CMEs. These CMEs are listed in Table 1. There is only one MC event in all of 2008 in the Lepping et al. (2011) list, and that is from 2008 December 16. This is the first event listed in Table 1. We emphasize that we would have considered earlier $2008 \mathrm{MCs}$ if there had been any. In five cases there are two MCs identified very close in time, which greatly complicates our interpretation. For two of these cases (\#20-21 and \#27-28 in Table 1) we associate the two MCs with two distinct CMEs seen erupting from the Sun. However, for three cases (\#8, \#11, and \#18) we only see a single CME, and we end up concluding that the one CME accounts for both MCs. This is why there are only 28 CMEs listed in Table 1 to account for the 31 MCs. There is more discussion of the multi-MC cases in the Appendix.

The CME start time listed in Table 1 corresponds to the time that expansion is first perceived close to the Sun in COR1 images. It should be noted that for some of the slow CMEs in our list, the expansion begins so gradually that there is ambiguity in defining a clear start time. The ICME arrival time at Earth is estimated from Wind data time-shifted to the bow shock nose, extracted from NASA/GSFC's OMNI database. The Wind measurements of proton density, velocity, and magnetic field are shown in Figure 1 for our 28 CMEs. The MC time intervals from Lepping et al. (2011, 2015) are indicated in the figure. However, in most cases inspection of the Wind data leads us to see the MC region as being just part of a larger ICME structure, due to high velocities, densities, and/or fields extending beyond the MC bounds. We estimate the ICME arrival time (to the nearest hour) based on the time of what we propose to be the initial density, velocity, and/or field increase of the broader ICME region. This assessment is in some cases unambiguous (e.g., events \#8 and \#18), but in other cases it is somewhat subjective (e.g., events \#13 and \#25). The assumed ICME arrival time is indicated in Figure 1 and listed in Table 1. Our events have travel times from the Sun to Earth that range from 1.85 days (\#26) to 7.29 days (\#4).

The $\phi_{l}$ and $\theta_{l}$ parameters in Table 1 indicate the magnetic cloud orientations suggested by Lepping et al. (2011, 2015). The analysis assumes the MC is a force-free FR with a cylindrical geometry, with $\left(\phi_{l}, \theta_{l}\right)$ indicating the attitude of the axis of the cylinder, in the direction of the axial magnetic field. Note that while Lepping et al. (2011, 2015) use GSE coordinates, we here convert to heliocentric Earth-ecliptic (HEE) coordinates, which are more natural for remote sensing studies of CMEs propagating from the Sun to Earth. In HEE coordinates, the x-axis points from the Sun towards Earth, the z-axis points from the Sun towards ecliptic north, and the y-axis points to the right from Earth's perspective (while facing the Sun), so $\tan \phi_{l}=y / x$ and $\tan \theta_{l}=z / \sqrt{x^{2}+y^{2}}$. The $Q_{l}$ parameter in Table 1 is an MC fit quality parameter from Lepping et al. (2011, 2015), with $Q_{l}=1,2$, or 3 indicating good, fair, or poor.

Partly to investigate systematic uncertainties in the MC parameters, we have performed an independent analysis of the MC events using the analysis approach described by Nieves-Chinchilla et al. (2016), relaxing the traditional force-free assumption used in the Lepping et al. (2011, 2015) fit. Two examples of the fits to the Wind magnetic field measurements are shown in Figure 2. The model assumes the same circular-cylindrical geometry assumptions as in the Lepping et al. $(2011,2015)$ fits. However, it does not assume any force distribution on the magnetic structure but makes assumptions for the axial and poloidal current density components. We assume the $\tau=1$ case described in equation (10) of Nieves-Chinchilla et al. (2016) (i.e., the axial field is assumed to go to zero at the outer edge of the FR).

A reconstruction is based on a multiple regression technique to infer the spacecraft trajectory using the Levenberg- 

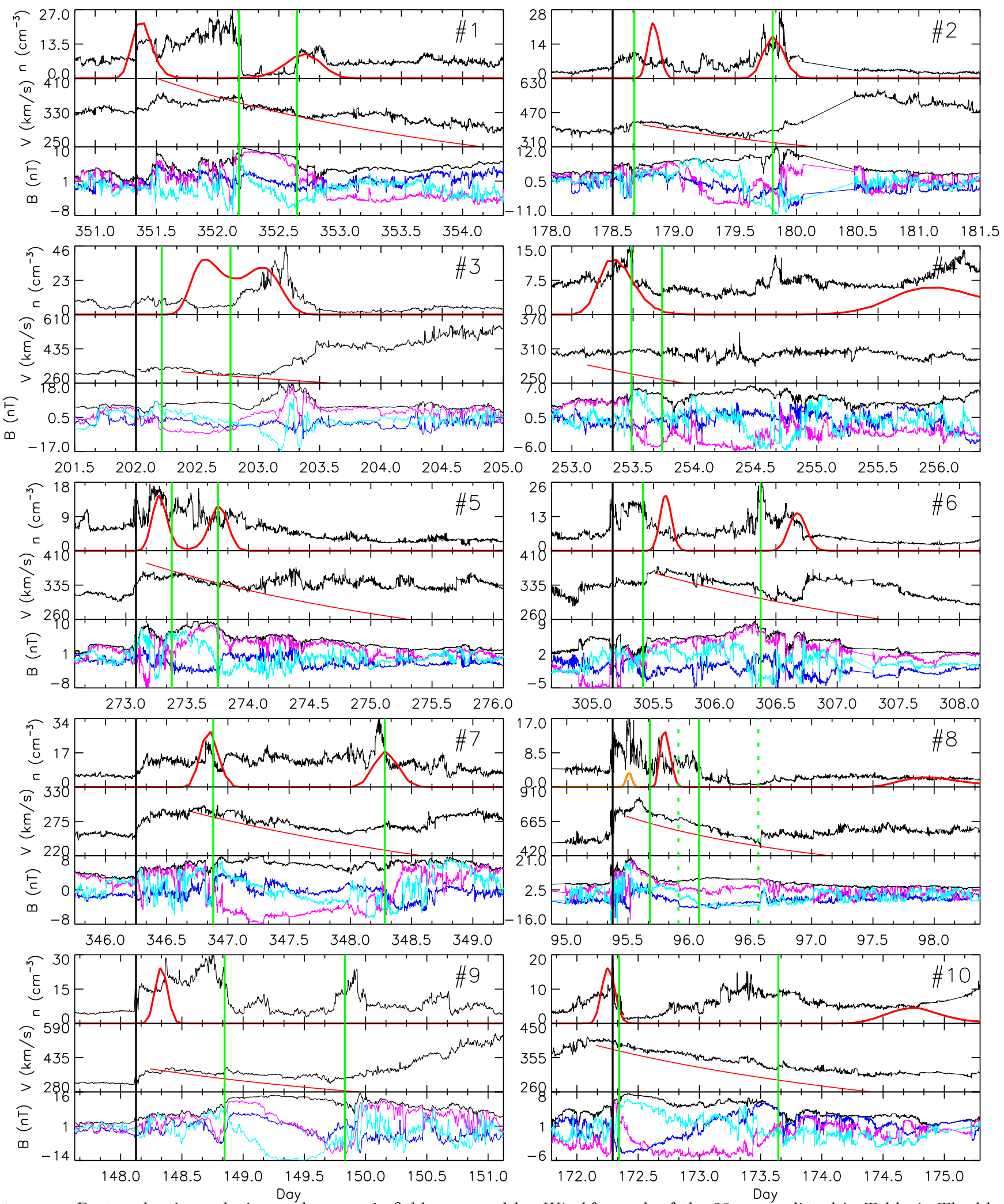

Figure 1. Proton density, velocity, and magnetic field measured by Wind for each of the 28 events listed in Table 1. The blue, purple, and light blue curves in the magnetic field panel are $B_{x}, B_{y}$, and $B_{z}$, while the black line is $B_{t o t}$. Vertical black lines indicate the estimated ICME arrival time. Vertical green lines indicate MC boundaries from Lepping et al. (2011, 2015). In five panels there are two MCs identified, the second with dotted lines. Red and orange lines show the density and velocity profiles predicted by the 3-D CME reconstruction described in Section 3, with red peaks in the density panel corresponding to the front and/or back of the FR, and orange peaks corresponding to a shock. In two panels (\#20-21, \#27-28), there are two CMEs shown, the second using dotted instead of solid lines. 

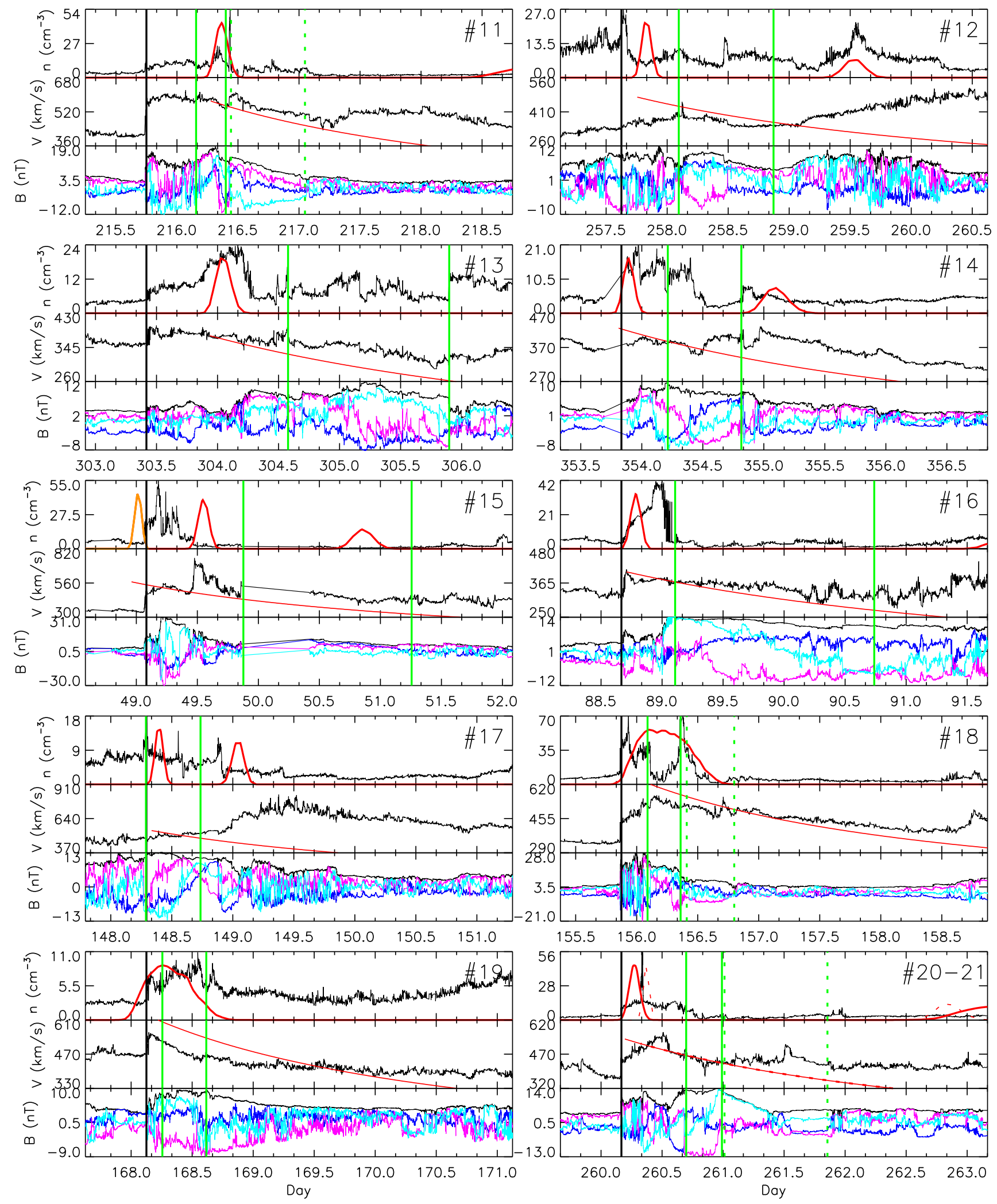

Figure 1. (continued) 

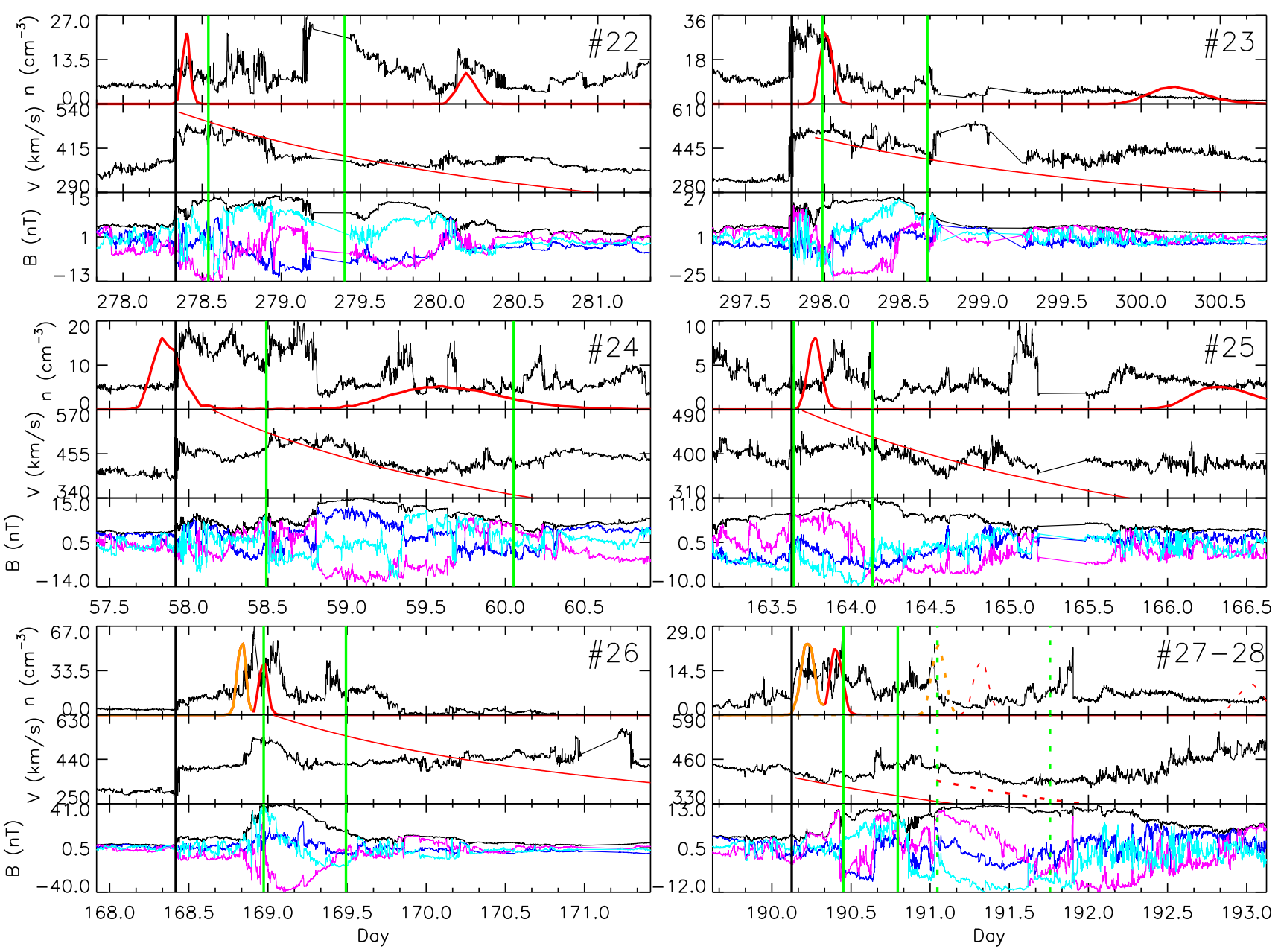

Figure 1. (continued)

Marquardt algorithm. The parameters are initialized using minimum variance for the orientation and magnetic field observations at the edge and center for the current density components. It is a semi-automatic iterative exercise going from the local flux-rope coordinate system to the spacecraft coordinate system to converge on the final parameters. The quality of the fittings are based on the correlation coefficient described by Nieves-Chinchilla et al. (2016). The $\phi_{n}$ and $\theta_{n}$ parameters in Table 1 indicate the resulting MC orientations, for the same boundaries as Lepping et al. (2011, 2015), and $Q_{n}$ is the fit quality indicator from this new analysis, which can be compared with the analogous $Q_{l}$ flag.

Finally, for all our events, we have looked for solar surface activity associated with each CME, particularly in EUV movies from the EUVI imagers on STEREO, as well as images from the Atmospheric Imaging Assembly (AIA) instrument on board the Solar Dynamics Observatory (SDO). We look for surface activity at the right time and location to connect with the CMEs in our list. Table 1 indicates which events are convincingly associated with significant flares in the EUV, and which are associated with clear filament eruptions. In the case of flares, the GOES flare strength designation is listed, if it has one. The start time of the activity is listed, which is naturally very close to the CME start time in all instances. The surface location of the activity in HEE coordinates is also listed in Table 1. For eight events in our sample, we find no surface activity at all that can be definitively associated with the CMEs.

Our sample of events overlaps with other studies that have connected CMEs observed by STEREO to ICMEs observed by spacecraft at 1 AU. Relevant surveys that we are aware of include Lugaz et al. (2012b), who studied ICMEs that impacted the STEREO spacecraft between 2008 and early 2010; Kilpua et al. (2012, 2014), who studied 1 AU travel times of 31 events from 2008-2010 and searched for solar sources of twenty 2009 ICME events observed at Earth; Möstl et al. (2014), who studied the kinematics of a sample of 24 CMEs from 2008-2012 that hit Wind or one of the STEREO spacecraft; and Wood et al. (2016b), who focused on STEREO observations of nine of the most geoeffective CMEs from the first half of solar cycle 24 . 

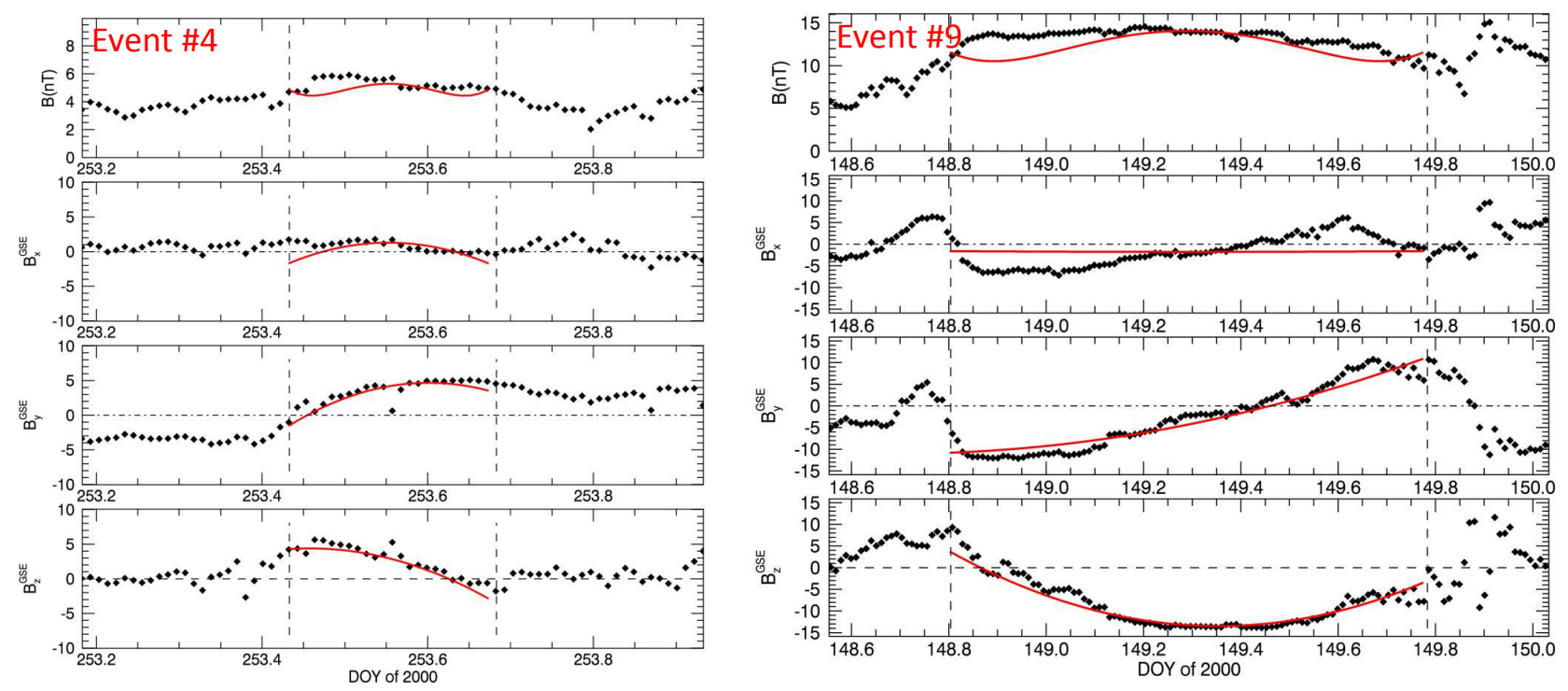

Figure 2. Two examples of magnetic cloud fits to Wind observations for events \#4 and \#9 in Table 1 , using the Nieves-Chinchilla et al. (2016) technique, leading to the $\left(\phi_{n}, \theta_{n}\right)$ MC orientation measurements quoted in Table 1.

Nevertheless, we believe our list represents the first complete STEREO survey of MC events in a multi-year time period. We verify that the majority of MCs detected at Earth are indeed ICMEs, as STEREO allows us to conclusively connect 31 out of $48(65 \%)$ of the 2008-2012 Wind MCs with CMEs. It is probable that many if not all of the remaining $17(35 \%)$ will also be CMEs, but ones that did not allow for a truly conclusive CME-MC connection. Our conservative approach of requiring CMEs to be trackable continuously from COR1 to HI2 will neglect cases of faint candidate CMEs that, for example, might be tracked into HI1, but are too faint to be followed into HI2. Obscuration by CIR fronts seen in HI2 (see, e.g., Wood et al. 2010b) or by other CME fronts could also play a role in hiding candidate CMEs. It is possible that some of the 17 unconnected MCs may not be ICMEs, but instead more quiescent solar wind structures, perhaps connected with CIRs or SIRs. It is worth noting that there is a tendency for the MCs successfully connected to ICMEs to be longer than the unconnected MCs, with the former having an average duration of $21.0 \mathrm{hr}$, and the latter having an average duration of $13.6 \mathrm{hr}$.

\section{RECONSTRUCTING CME KINEMATICS AND MORPHOLOGY FROM STEREO IMAGES}

For each event in Table 1, we use STEREO and SOHO/LASCO images to measure CME kinematics and morphology, operating within the context of the flux rope paradigm for CME structure. As an example, Figure 3 shows a sequence of images following the 2012 February 24 CME (event \#24) from the Sun to near 1 AU. The COR1 and COR2 images are displayed in a base-difference format, with an image from before the CME subtracted from each CME image. The LASCO/C3, HI1, and HI2 images are shown in a running-difference format, with the previous image subtracted from each image. Movies showing the sequence of STEREO and SOHO/LASCO images of all events are available in the online version of this article.

Our techniques for 3-D CME reconstruction are already well established in the literature (Wood \& Howard 2009; Wood et al. 2009, 2010a, 2011, 2012a,b, 2016b), though we briefly describe them again here. In our approach, the kinematic and morphological analyses are performed separately, but there is interdependence between the two. Thus, in practice there is iteration between the kinematic and morphological parts of the analysis to reach a self-consistent final solution.

\subsection{Kinematic Analysis}

We first focus on the kinematic analysis, which begins with measurements of elongation angles of the CME leading edge from Sun-center $(\epsilon)$. These measurements are made from a single viewpoint, either STEREO-A or -B, depending on which image set provides the clearest tracking of the CME front from COR1 to HI2. The elongation angles must be converted to actual distances from Sun center $(r)$, but this conversion requires geometric assumptions about the shape of the CME leading edge. If the CME is assumed to be very narrow,

$$
r=\frac{d \sin \epsilon}{\sin (\epsilon+\phi)}
$$



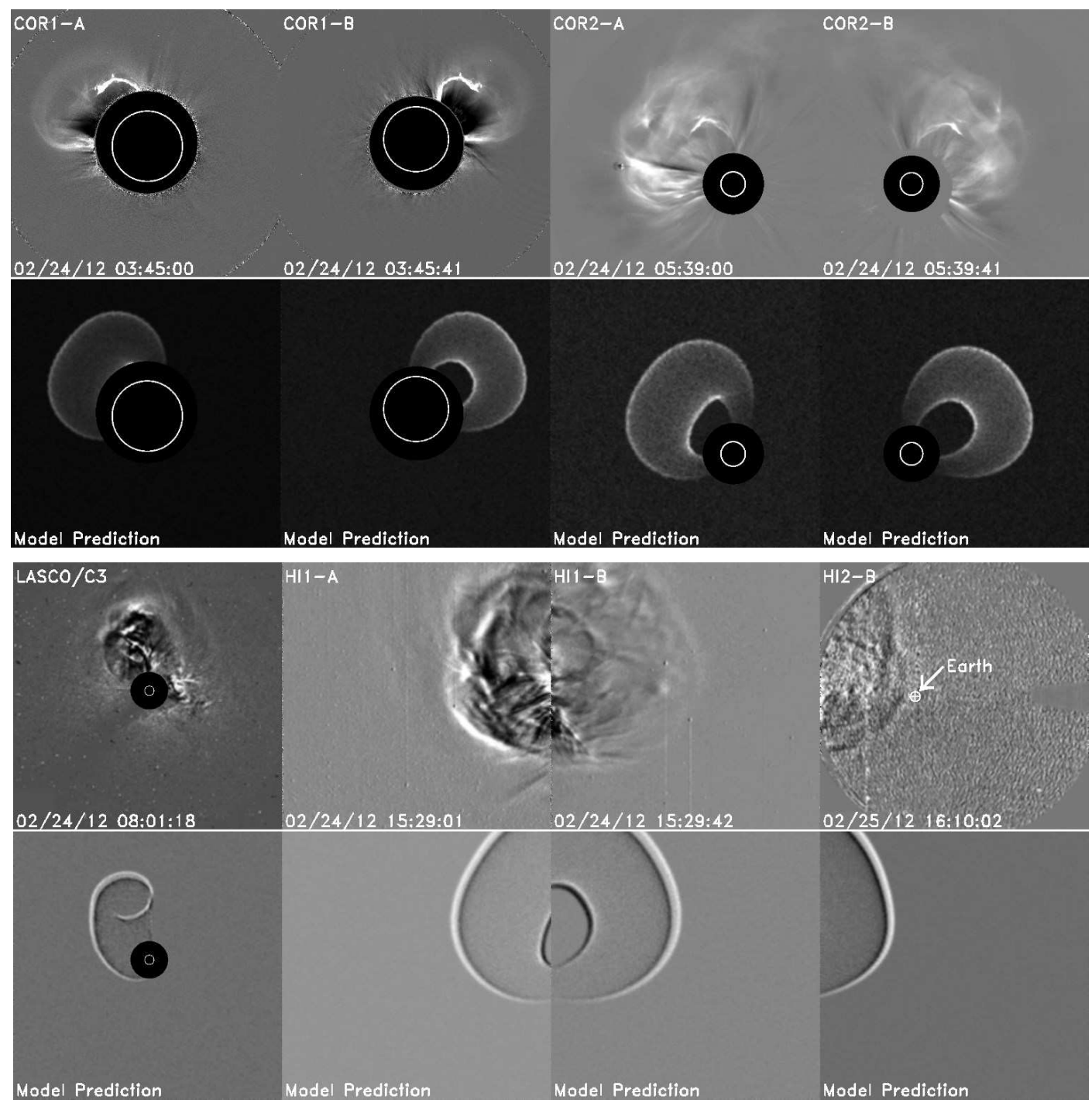

Figure 3. A sequence of 8 STEREO and SOHO/LASCO images of a CME from 2012 February 24 (event \#24), in chronological order. Below each image is a synthetic image computed from the $3-\mathrm{D}$ reconstruction of this event.

where $d$ is the distance from the observer to the Sun and $\phi$ is the angle between the CME trajectory and the observer's line of sight to the Sun (Kahler \& Webb 2007; Sheeley et al. 2008). This can be referred to as the "Fixed- $\phi$ " approximation. Another common assumption is that the CME front can be approximated as a sphere centered halfway between the Sun and the leading edge, leading to the so-called "Harmonic Mean" approximation (Lugaz et al. 2009),

$$
r=\frac{2 d \sin \epsilon}{1+\sin (\epsilon+\phi)}
$$

More complex assumptions about CME front geometry lead to relations with more free parameters (e.g., Davies et al. 2013), but we will here consider only the above two relations.

There are three distinct constraints on the trajectory direction quantified by $\phi$ in equations (1) and (2), which can be called the "stereoscopic constraint," the "reasonable kinematics constraint," and the "Earth arrival time constraint." The first is by far the most important, which is provided by comparing the angular extent of the CME from the perspectives of STEREO-A and -B. This assessment comes from the morphological analysis described in Section 3.2, involving reconstruction of the CME's shape as well as its trajectory direction. The second constraint involves the assumption that at large distances from the Sun the CME should trend towards a constant terminal speed. In particular, there should be no implausible sudden accelerations or decelerations far from the Sun. The final constraint, which depends on both the kinematic and morphological parts of the analysis, is that the CME must hit Earth at the observed time (see Table 1). 

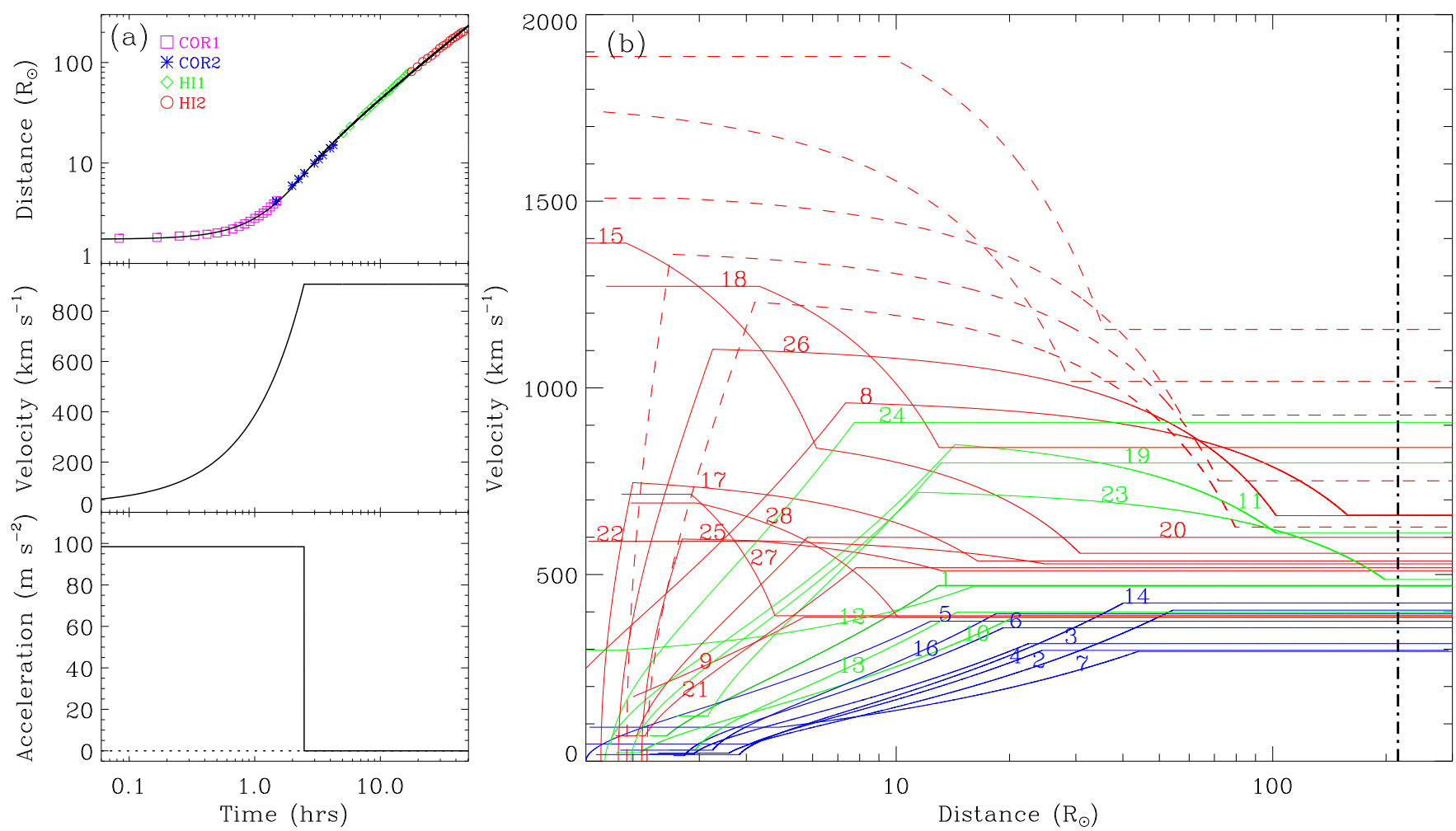

Figure 4. (a) Kinematic model for event \#24 in Table 1, with the top panel showing distance versus time measurements for the leading edge of the CME, from STEREO-B for this particular event. These measurements are fitted with a simple two-phase kinematic model, yielding the solid line fit to the data, and the velocity and acceleration profiles shown in the lower panels. (b) Velocity versus distance profiles for the leading edges of all 28 CMEs listed in Table 1, with line color indicating solar surface activity associated with the events. Red, green, and blue indicates association with flares, filament eruptions (but no flares), and no surface activity at all, respectively. The red dashed lines are additional kinematic profiles from Wood et al. (2016b), for five geoeffective events not perceived as MCs at Earth. The dot-dashed line indicates 1 AU.

We experiment with both the "Harmonic Mean" and "Fixed- $\phi$ " approximations, and use whichever is most successful at finding a $\phi$ value that simultaneously addresses all three constraints mentioned above. A previous analysis of an event from 2008 June 1 event provides an excellent example of a case where the $\phi$ value inferred from the "stereoscopic constraint" yields a far more reasonable kinematic profile (i.e., the "reasonable kinematics constraint") when "Harmonic Mean" is assumed than when "Fixed- $\phi$ " is used (see Figure 2 in Wood et al. 2010a). Our survey now allows us to say that this is indeed the norm, and that the "Harmonic Mean" approximation is usually far more successful in this regard than "Fixed- $\phi . "$ There are only four cases (events \#5, \#13, \#17, and \#25) where we end up preferring "Fixed- $\phi . "$

Once the CME leading edge distances $(r)$ are established, we can study CME kinematics, starting with plots of $r$ versus time. The top panel of Figure 4(a) shows such a plot for event \#24. From such measurements we seek to infer velocity and acceleration profiles. For that purpose, we use a simple kinematic model that we have used many times in the past (Wood \& Howard 2009; Wood et al. 2009, 2010a, 2011, 2012a,b, 2016b), which divides the CME travel time into $2-4$ phases of constant velocity and/or acceleration (or deceleration). Decisions on the type and number of phases are made after inspecting velocity-versus-time profiles computed point-by-point. The kinematic model for event \#24 in Figure 4(a) is particularly simple, with only two phases: a constant acceleration phase, followed by a constant velocity phase.

Assuming 1\%, 2\%, and 3\% uncertainties for the distance measurements from COR1/COR2, HI1, and HI2, respectively (e.g., Wood \& Howard 2009), we use a $\chi^{2}$ minimization routine to find the best fit to the distance measurements (Bevington \& Robinson 1992). For event \#24, the top panel of Figure 4(a) shows the best fit to the data, and the panels below show the corresponding velocity and acceleration profiles. For this two-phase fit there are only three free parameters: a starting height, the acceleration for the first phase, and a time for the end of that phase.

Figure 4(b) shows the velocity versus distance profiles of all $28 \mathrm{CMEs}$ in our sample. From these curves we can infer a peak velocity $\left(V_{\max }\right)$ and a terminal speed far from the Sun $\left(V_{\text {term }}\right)$. These values are listed in Table 2 , along with a number of morphological parameters that will be described in Section 3.2. Our MC-selected CME sample includes only 
Table 2. CME Measurements

\begin{tabular}{|c|c|c|c|c|c|c|c|c|c|c|c|c|c|}
\hline ID & $\begin{array}{c}\lambda_{s}{ }^{a} \\
(\mathrm{deg})\end{array}$ & $\begin{array}{c}\beta_{s}^{a} \\
(\operatorname{deg})\end{array}$ & $\begin{array}{c}\gamma_{s}^{b} \\
(\operatorname{deg})\end{array}$ & $\begin{array}{c}\mathrm{FWHM}_{s}{ }^{c} \\
(\mathrm{deg})\end{array}$ & $\Lambda_{s}^{d}$ & $\eta_{s}^{e}$ & $\alpha_{s}^{f}$ & $\mathrm{Q}_{s}{ }^{g}$ & $\begin{array}{c}\mathrm{V}_{\max }{ }^{h} \\
\left(\mathrm{~km} \mathrm{~s}^{-1}\right)\end{array}$ & $\begin{array}{c}\mathrm{V}_{\text {term }}{ }^{h} \\
\left(\mathrm{~km} \mathrm{~s}^{-1}\right)\end{array}$ & $\begin{array}{l}D_{s t}{ }^{i} \\
(\mathrm{nT})\end{array}$ & $\begin{array}{c}\phi_{s}^{j} \\
(\operatorname{deg})\end{array}$ & $\begin{array}{c}\theta_{s}^{j} \\
(\operatorname{deg}) \\
\end{array}$ \\
\hline 1 & 9 & 10 & -60 & 77.4 & 0.25 & 1.1 & 2.5 & 4 & 470.4 & 470.4 & -15 & 102.9 & -51.1 \\
\hline 2 & 13 & -10 & -20 & 70.8 & 0.10 & 2.31 & 8.0 & 4 & 404.4 & 404.4 & -28 & 70.1 & -20.8 \\
\hline 3 & -9 & -6 & -8 & 62.0 & 0.07 & 1.0 & 8.0 & 2 & 315.0 & 315.0 & -83 & 100.0 & -5.6 \\
\hline 4 & -25 & -5 & -5 & 76.3 & 0.18 & 1.6 & 6.0 & 3 & 296.9 & 296.9 & -14 & 117.7 & 0.9 \\
\hline 5 & 1 & -2 & -10 & 41.5 & 0.06 & 1.5 & 4.0 & 1 & 374.7 & 374.7 & -9 & 87.1 & -10.0 \\
\hline 6 & 8 & 1 & -4 & 41.5 & 0.10 & 1.0 & 4.0 & 2 & 357.6 & 357.6 & -10 & 73.2 & -3.1 \\
\hline 7 & 9 & -5 & -40 & 52.1 & 0.12 & 1.5 & 3.0 & 3 & 294.2 & 294.2 & -2 & 68.9 & -33.9 \\
\hline 8 & 3 & -16 & -80 & 59.9 & 0.21 & 1.6 & 4.0 & 3 & 959.9 & 659.9 & -81 & 12.4 & -43.6 \\
\hline 9 & 12 & 3 & 50 & 76.3 & 0.31 & 1.0 & 6.0 & 3 & 385.9 & 385.9 & -80 & 71.0 & 40.3 \\
\hline 10 & -11 & 10 & -25 & 64.5 & 0.17 & 1.6 & 4.0 & 3 & 391.0 & 391.0 & -11 & 144.6 & -15.7 \\
\hline 11 & -6 & 20 & -40 & 80.5 & 0.30 & 1.3 & 3.0 & 2 & 848.1 & 612.0 & -74 & 128.4 & -35.9 \\
\hline 12 & -8 & 15 & -35 & 104.3 & 0.20 & 1.7 & 5.0 & 4 & 468.4 & 468.4 & -25 & 93.9 & -34.3 \\
\hline 13 & 9 & -30 & -65 & 111.9 & 0.22 & 1.4 & 3.5 & 4 & 399.5 & 399.5 & -11 & 20.0 & -39.6 \\
\hline 14 & 10 & -10 & -25 & 73.7 & 0.12 & 2.0 & 4.0 & 3 & 423.8 & 423.8 & -35 & 61.8 & -23.4 \\
\hline 15 & -8 & -10 & 45 & 92.0 & 0.15 & 2.0 & 2.5 & 3 & 1387.8 & 556.8 & -31 & 114.9 & 38.3 \\
\hline 16 & -11 & 5 & -25 & 82.2 & 0.25 & 1.4 & 4.5 & 3 & 395.2 & 395.2 & -4 & 101.6 & -23.7 \\
\hline 17 & 1 & -5 & -80 & 62.8 & 0.10 & 1.5 & 6.0 & 4 & 746.2 & 536.3 & -80 & 72.7 & -66.9 \\
\hline 18 & -25 & -5 & -40 & 83.0 & 0.31 & 1.1 & 4.0 & 2 & 1272.0 & 840.3 & -45 & 108.0 & -27.9 \\
\hline 19 & -39 & -5 & 35 & 119.8 & 0.18 & 1.6 & 4.0 & 4 & 798.9 & 798.9 & -14 & 122.6 & 17.2 \\
\hline 20 & 23 & 10 & 35 & 85.3 & 0.23 & 1.4 & 3.0 & 3 & 599.6 & 599.6 & -69 & 41.7 & 19.0 \\
\hline 21 & -2 & 15 & -75 & 73.7 & 0.25 & 1.3 & 4.0 & 3 & 518.2 & 518.2 & -69 & 142.6 & 13.5 \\
\hline 22 & -1 & -3 & -50 & 59.0 & 0.21 & 1.0 & 5.0 & 3 & 589.1 & 510.0 & -43 & 89.8 & -43.7 \\
\hline 23 & -5 & 25 & -60 & 98.7 & 0.19 & 1.5 & 6.0 & 3 & 720.3 & 486.8 & -147 & 147.7 & 12.8 \\
\hline 24 & -26 & 35 & -73 & 118.4 & 0.29 & 1.25 & 3.0 & 5 & 907.6 & 907.6 & -48 & 151.5 & -35.7 \\
\hline 25 & 2 & -20 & -80 & 75.8 & 0.19 & 1.6 & 3.0 & 3 & 595.0 & 528.5 & -51 & 9.9 & -43.1 \\
\hline 26 & -3 & -20 & -10 & 113.7 & 0.43 & 1.6 & 3.0 & 2 & 1103.5 & 657.7 & -71 & 90.0 & -9.7 \\
\hline 27 & -1 & -10 & -85 & 66.3 & 0.25 & 1.0 & 3.0 & 2 & 715.0 & 389.7 & -68 & 9.7 & -53.1 \\
\hline 28 & -1 & 10 & 30 & 83.0 & 0.19 & 1.3 & 4.0 & 2 & 691.8 & 385.6 & -68 & 89.5 & 28.6 \\
\hline
\end{tabular}

NotE - ${ }^{a}$ Longitude and latitude of central FR trajectory, in HEE coordinates. ${ }^{b}$ Tilt angle of FR $\left(0^{\circ}\right.$ is an E-W orientation). ${ }^{c} \mathrm{FR}$ angular full width at half-maximum. ${ }^{d} \mathrm{FR}$ aspect ratio (apex radius divided by distance from Sun). ${ }^{e}$ Ellipticity of FR cross section. ${ }^{f}$ Parameter defining shape of FR leading edge. ${ }^{g}$ Quality flag $(1-5)$ assessing the evidence for an FR shape $(1=$ no evidence, $5=$ strong evidence). ${ }^{h}$ Peak and terminal CME speeds. ${ }^{i}$ Minimum $D_{s t}$ value, as a measure of geoeffectiveness. ${ }^{j}$ FR axis direction seen by Earth, in HEE coordinates.

three events that reach $V_{\max }>1000 \mathrm{~km} \mathrm{~s}^{-1}$, and none with $V_{\max }>1500 \mathrm{~km} \mathrm{~s}^{-1}$. In order to increase the number of fast CMEs in our sample, we have in Figure 4(b) added kinematic profiles for five additional events from Wood et al. (2016b), who studied nine geoeffective CMEs, performing kinematic and morphological reconstructions for eight of them based on STEREO images. Three of these eight are already in our sample (events \#8, \#18, and \#23), but five are not. These events, with initiation dates of 2011 August 3, 2011 September 24, 2012 March 7, 2013 October 29, and 2014 February 25, all have $V_{\max }>1000 \mathrm{~km} \mathrm{~s}^{-1}$ but were not perceived as MCs at Earth, despite being very geoeffective. These are the five events whose kinematic profiles are shown as dashed lines in Figure 4(b).

Figure 4(b) divides the CMEs into three categories, based on what solar surface activity is associated with the events. We will refer to events associated with flares as "group 1," events associated with filament eruptions but no flares "group 2," and events with no surface activity "group 3." If the five extra geoeffective events are included, groups 1, 2 and 3 include 17, 8 , and 8 events, respectively. The group 3 events are all slow, with peak speeds of $V_{\max }=294.2-423.8 \mathrm{~km} \mathrm{~s}^{-1}$. The group 1 CMEs are all faster than this, with one exception (event \#9). Group 2 


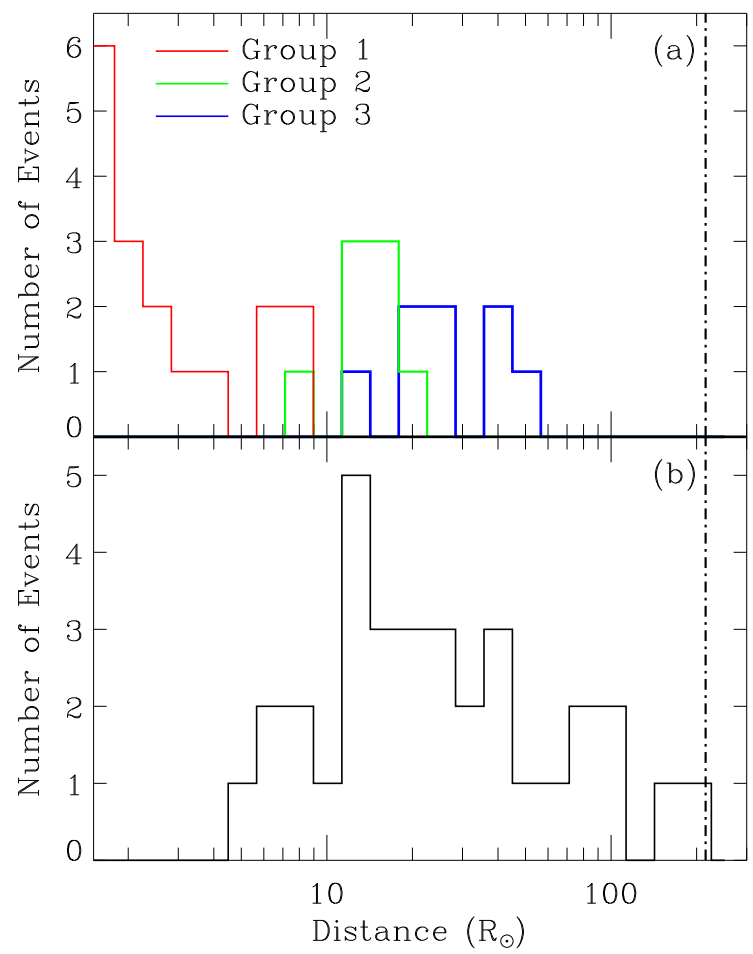

Figure 5. (a) Distribution of heights at which CMEs first reach their peak velocities for group 1 (flare-associated CMEs), group 2 (filament eruption associated CMEs), and group 3 (no surface activity). (b) Distribution of heights at which CMEs reach their final terminal speeds. The vertical dot-dashed line indicates $1 \mathrm{AU}$.

is intermediate, with half being slow $\left(V_{\max }<500 \mathrm{~km} \mathrm{~s}^{-1}\right)$ and half relatively fast, though none reach $V_{\max }>1000$ $\mathrm{km} \mathrm{s}^{-1}$.

Another very clear distinction in the kinematic behavior of these three groups regards the height at which the peak velocity is reached. This is shown explicitly in Figure 5(a). We compute the average and standard deviation of this height for all three categories, and find $R=3.2 \pm 2.2 \mathrm{R}_{\odot}, R=13.9 \pm 3.8 \mathrm{R}_{\odot}$, and $R=29.4 \pm 14.8 \mathrm{R}_{\odot}$ for groups 1 , 2 , and 3, respectively. Note that for group 1, EUVI images of the CME fronts closer to the solar surface would be required to precisely assess the height when peak velocity is reached, as about half of these events are already at or near their peak speeds when they enter the COR1 field of view at $R \approx 1.5 \mathrm{R}_{\odot}$.

As shown in Figure 4(b), all but two events that reach $V_{\max }>600 \mathrm{~km} \mathrm{~s}^{-1}$ subsequently decelerate, the example in Figure 4(a) being one of the two exceptions. The deceleration is presumably due to drag forces induced by collision with the slower ambient solar wind. For a few CMEs (e.g., events \#15 and \#27) deceleration seems to happen very early at $R<10 \mathrm{R}_{\odot}$, but in most cases the deceleration happens mostly further out. Figure 5(b) shows the distributions of heights at which CMEs reach their final terminal speeds. For fast events, this would generally be where deceleration ceases, while for slow events this would be where acceleration to the terminal speed stops. The CMEs reach their final speeds at heights ranging from $R=4.8-198.7 \mathrm{R}_{\odot}$. Half of our events have reached their final speeds by $R=20 \mathrm{R}_{\odot}$, while $80 \%$ have reached it by $R=0.3 \mathrm{AU}\left(64.5 \mathrm{R}_{\odot}\right)$. Finally, it should be emphasized that contrary to the velocity profiles in Figure 4(b), it is likely that many CMEs still exhibit some degree of deceleration at 1 AU. Our simple, multi-phase kinematic modeling approach neglects this by invariably assuming a final phase of constant velocity. Quantifying residual CME deceleration at $1 \mathrm{AU}$ would ideally require observational tracking of the CMEs well beyond $1 \mathrm{AU}$.

\subsection{Morphological Analysis}

For the morphological part of our analysis, we use a parametrized prescription for generating 3-D FR shapes. For each such shape, a 3-D density distribution is created with density placed on the boundary of the FR shape, and these density cubes are then used to compute synthetic images for comparison with real ones. Parameters are varied until agreement with the data is maximized. Probably the most widely used prescription for FR shape generation, and comparison with STEREO imagery, is that of Thernisien et al. (2006, 2009), for which there is a user friendly IDL tool. We instead use a procedure first described by Wood \& Howard (2009), and which has been subsequently used in 
many published analyses (Wood et al. 2010a, 2011, 2012a,b, 2016b).

The inner and outer edges of the FR are defined in polar coordinates with the equation

$$
r(\theta)=r_{\max } \exp \left(-\frac{1}{2}\left|\frac{\theta}{\sigma}\right|^{\alpha}\right) \text {. }
$$

This is a polar Gaussian if $\alpha=2$. Higher $\alpha$ values create loops with flatter, less rounded leading edges. The $\sigma$ parameter determines the widths of the loops, which can also be described by a full-width-at-half-maximum,

$$
F W H M_{s}=2(1.386)^{1 / \alpha} \sigma .
$$

As described by Wood \& Howard (2009), the two 2-D loops defining the inner and outer edges of the FR are used to create a 3-D FR by first creating a sequence of lines normal to the inner edge, extending to the outer edge. The midpoints of these lines define the central axis of the FR. A 3-D FR can be created by assuming circular cross sections for the flux rope in all the planes defined by the normal lines. By stretching the FR in the direction perpendicular to the FR creation plane, an FR can be created with an arbitrary ellipticity, with the parameter $\eta_{s}$ describing the ratio of FR channel width perpendicular to the FR plane to its width within the FR plane.

The two main advantages of this FR shape prescription to that of Thernisien et al. (2006, 2009) are the ability to assume elliptical FR channels, and the ability (by increasing $\alpha$ ) to assume FRs with flattened fronts. Note that Janvier et al. $(2013,2014)$ have developed prescriptions for describing CME front shapes that could easily replace equation (3), and then be used to create 3-D FR shapes. With straighter, more cone-like sides, these prescriptions may be better at describing the shapes of the trailing parts of CME fronts Janvier et al. (2013).

There are three parameters describing how to orient the 3-D FRs. A longitude and latitude in HEE coordinates $\left(\lambda_{s}, \beta_{s}\right)$ describe the trajectory direction of the center of the FR. A tilt angle $\gamma_{s}$ describes how the FR axis is oriented relative to the ecliptic plane, with $\gamma_{s}=0^{\circ}$ corresponding to an east-west orientation, parallel to the ecliptic, and $\gamma_{s}= \pm 90^{\circ}$ corresponding to a north-south orientation, perpendicular to the ecliptic. A density cube containing a 3-D FR described by the above parameters is created, placing density only on the surface of the FR, assuming a Gaussian density profile normal to the surface. Wood \& Howard (2009) list a number of parameters describing how density is placed on the FR surface, but these parameters are not of much interest for our purposes, so we do not discuss them here.

In order to confront the assumed FR shape with data, we first expand it to the appropriate size using the kinematic model described in Section 3.1. We assume that the same CME structure applies at all times during the CME's expansion outwards. In other words, we assume purely radial and self-similar expansion. In certain past analyses we have relaxed these assumptions and allowed for time-dependent evolution of CME morphology Wood et al. (2010a, 2012a,b), but this is too time consuming for a survey of many events. Our impression is that self-similar expansion is a decent, albeit not perfect, approximation for CMEs expanding into the interplanetary medium.

Synthetic images are then generated from the density cube using a white-light rendering routine to perform the necessary calculations of Thomson scattering within the density cube (Billings 1966; Thernisien et al. 2006). In this way, we can generate synthetic images of the CME at any time and from any perspective that we want, for comparison with images from STEREO-A, STEREO-B, and SOHO/LASCO. Parameters are adjusted to maximize agreement between the real and synthetic images. This is done by trial-and-error, and subjective judgment is used to decide what is the best fit. Figure 3 shows synthetic images computed in this manner for the 2012 February 24 CME (event \#24). Considering the number of images we have to work with, we in practice compare real and synthetic movies of the images. Movies comparing the real and synthetic STEREO and SOHO/LASCO images are available in the online version of this article, for all the events in our sample.

A secondary consideration in identifying the best fit parameters is the agreement between the observed and predicted Earth arrival time. This is an issue for the morphological as well as the kinematic part of the analysis, as changing the CME's shape or trajectory direction can affect the Earth arrival time as much as changing the kinematics of the CME leading edge. The density and velocity profiles predicted by our best models are compared with the Wind data in Figure 1. Since we only place density on the surfaces of our FRs, the very simplistic model prediction is for two density peaks, when Wind enters and exits the FR. (In some cases the exit peak is beyond the time range displayed in Figure 1.) The predicted velocity profile is always one of decreasing velocity, which is a characteristic of self-similar expansion, where velocity within the CME must be proportional to distance from the Sun for the CME to stay the same shape. These velocity decreases are similar to those that are generally observed within the MC time range, which will be discussed further in Section 5.3.

Figure 6 shows two different visualizations illustrating the 3-D CME reconstructions, the right one being a picture 

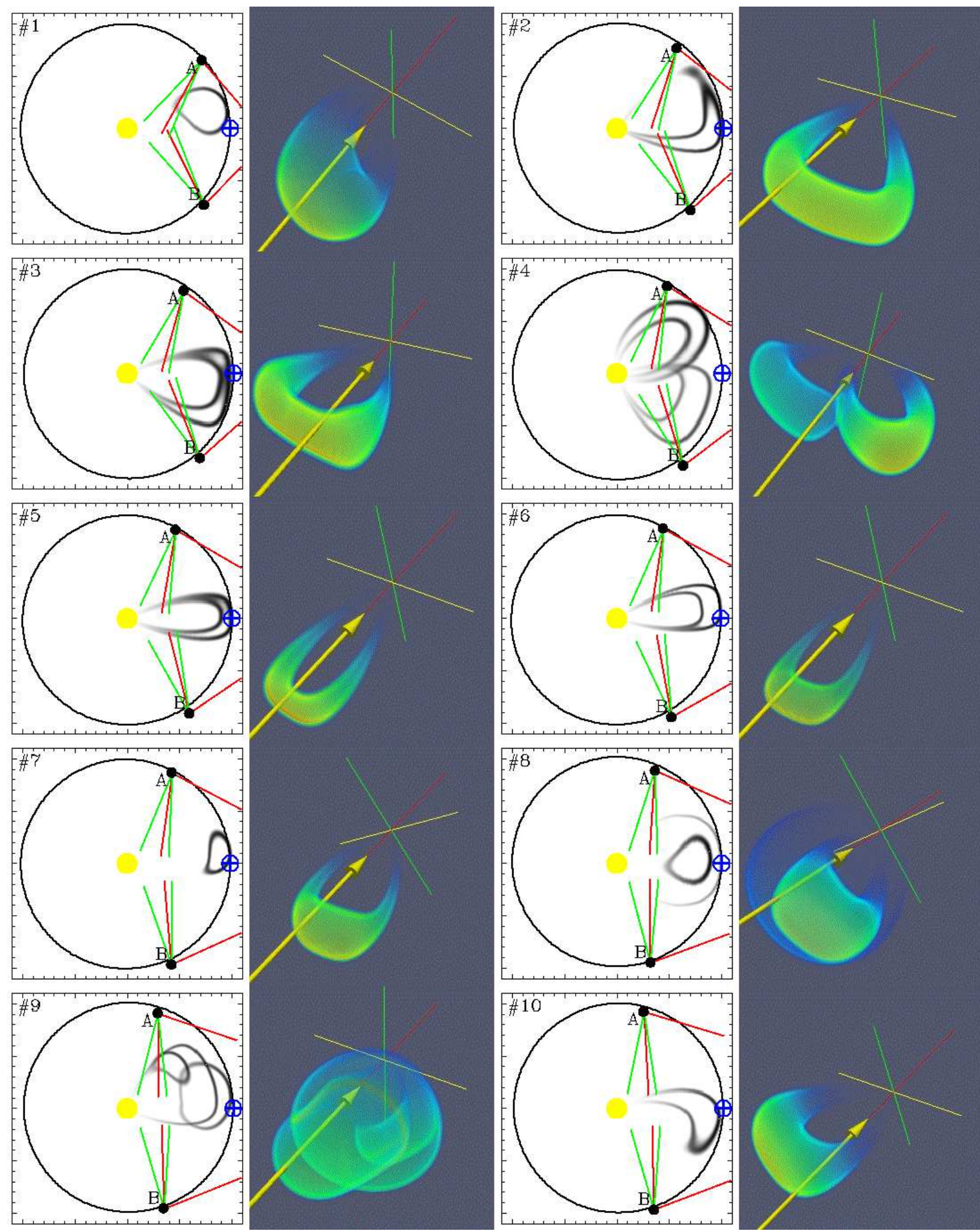

Figure 6. Two illustrations of the CME reconstruction are provided for each of the 28 events listed in Table 1. The left panels are ecliptic plane maps showing the positions of Earth, STEREO-A, and STEREO-B in HEE coordinates, with the greyscale image being the ecliptic plane slice through the 3-D CME reconstruction shown in the right panel. The green and red lines show explicitly the HI1 and HI2 fields of view. In the right panel, the x-, y-, and z-axes are in red, yellow, and green, respectively. The xy-plane is the ecliptic, with the x-axis defined by the Sun-Earth line, and the positive z-axis direction being ecliptic north (always up in each figure). The yellow arrow indicates the Earth's path through the CME structure. Note that many of the reconstructions actually include multiple CMEs (see text for details). 

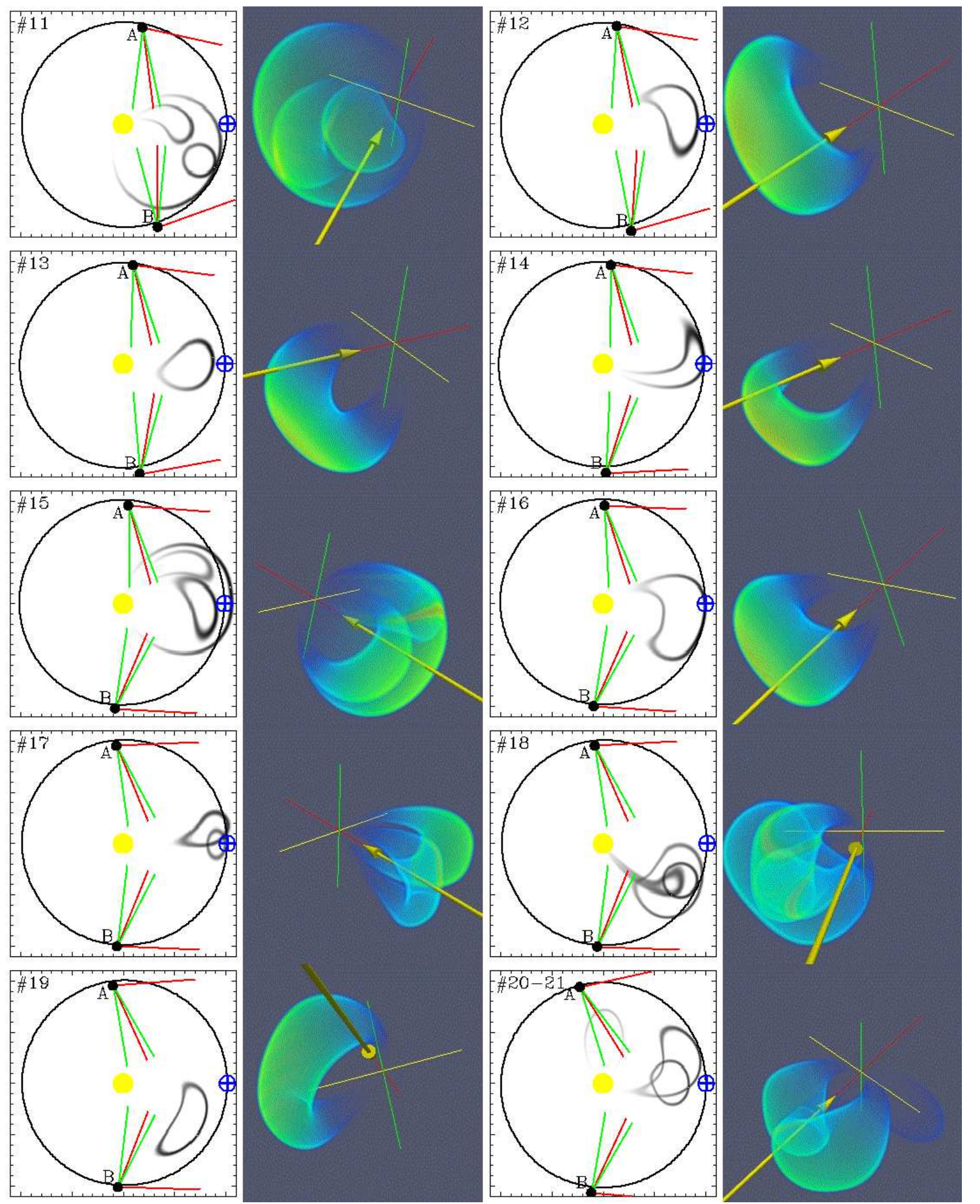

Figure 6. (continued) 

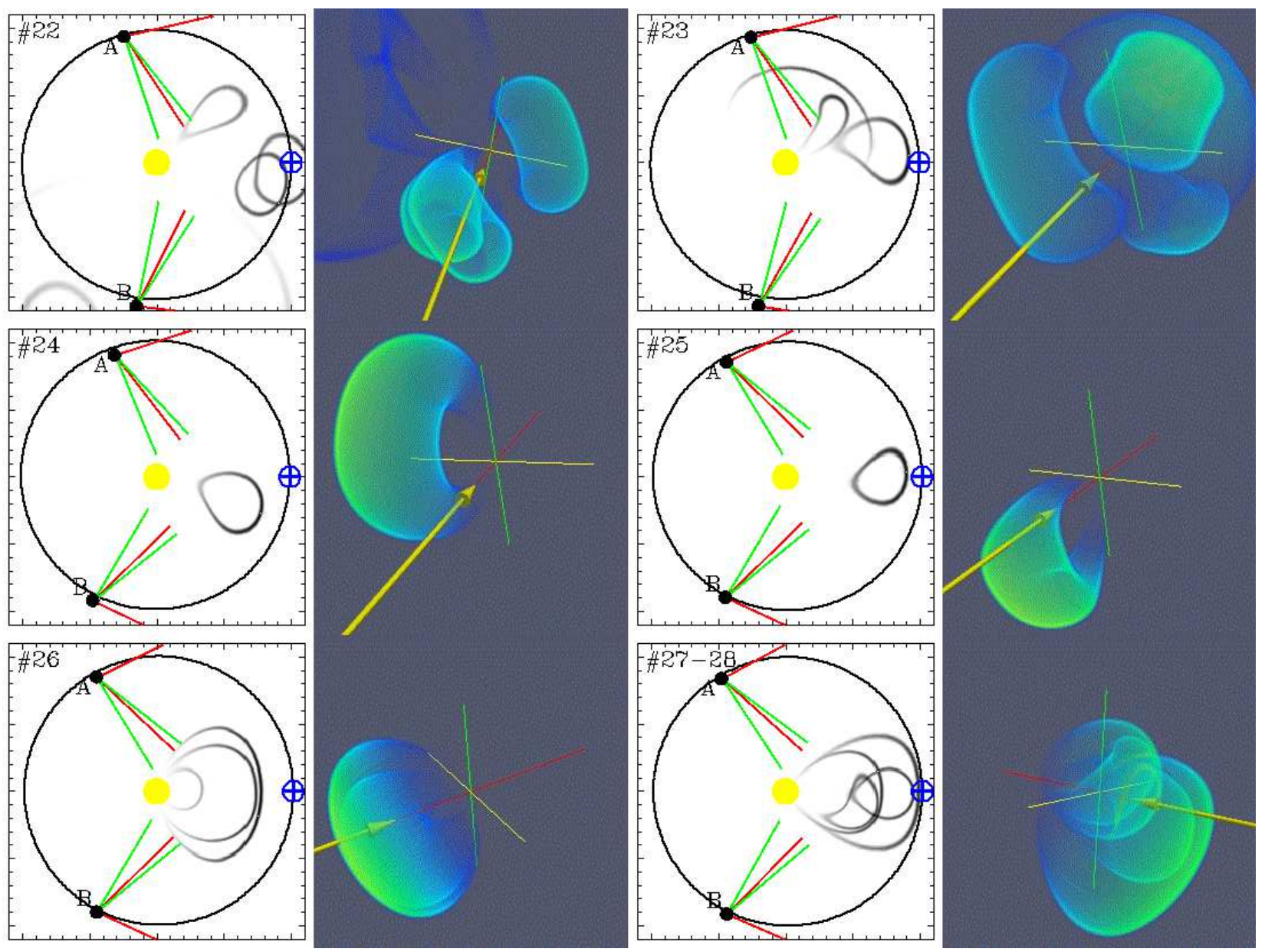

Figure 6. (continued)

of the 3-D structure in HEE coordinates, with an arrow indicating the Earth's path through it as the CME expands outwards, and the left one being a map in the ecliptic plane displaying a slice through the 3-D density cube in that plane. The ecliptic plane maps indicate the STEREO-A, STEREO-B, and SOHO/LASCO (near Earth) viewing geometry for each event, with the HI1 and HI2 fields of view explicitly displayed for both STEREO-A and -B. Columns 2-8 in Table 2 list the best-fit parameters of the FR structures. Note that we are using "s" subscripts for all morphological parameters derived from empirical analysis of STEREO and SOHO/LASCO images. The $\Lambda_{s}$ parameter is an aspect ratio dividing the FR apex radius by its distance from the Sun. A larger $\Lambda_{s}$ corresponds to a fatter FR. The $\alpha_{s}$ parameter in Table 2, from equation (3), is the one for the leading edge of the FR and not the trailing edge, which can be different.

The images of some of our faster events show a front out ahead of what we interpret as the FR ejecta, which is naturally interpreted as a shock. If this shock is sufficiently bright and trackable for a long distance from the Sun, we model it as well as the FR, as we have done for past events (e.g., Wood \& Howard 2009). To model a shock front shape, equation (3) is used to create a 2-D loop and then that loop is rotated about its center to create a 3-D lobular front. As in the FR modeling, the various parameters are adjusted to maximize agreement with the images. We ended up including shocks in the 3 -D reconstructions of only five of the events in the Table 1 sample, specifically events \#8, $\# 15, \# 26, \# 27$, and \#28 (see Figure 6). It is important to note that in the kinematic analysis in Section 3.1 we always seek to follow what we perceive as the top of the FR ejecta, and not the shock, although the two can be difficult to distinguish sometimes, particularly in HI1/HI2.

It is not uncommon for multiple CMEs to be visible simultaneously in the STEREO and SOHO/LASCO images. In some cases, the other $\mathrm{CME}$ fronts are distinguishable from the one of interest to an extent that we simply ignore the other CMEs, but in some instances we found it desirable to reconstruct multiple CMEs to ensure that we are correctly identifying the one that hits Earth and is responsible for the MC. Thus, in Figure 6 multiple CME structures 
are sometimes seen. In most cases the extra CMEs that are modeled are not Earth-directed, and are therefore distinguishable from the MC-related events of interest in Figure 6. But there are some exceptions (e.g., event \#22), and there are also the two cases of two separate CMEs interpreted as being responsible for two adjacent MC events in the Wind data (events \#20-21 and \#27-28). Discussion of individual events is provided in an Appendix, which should be consulted for more details of each particular analysis.

The $Q_{s}$ value listed in Table 2 is a quality flag indicating our assessment of the level of evidence for an FR structure within each CME, with $Q_{s}=1$ corresponding to no evidence for an FR shape, and $Q_{s}=5$ corresponding to very strong evidence in favor of an FR shape. Event \#5 is the only one at the negative $Q_{s}=1$ extreme. This is a faint event that is practically invisible in LASCO and COR1. It appears narrow and jet-like in the STEREO images at later times, and it is simply too faint to provide any indication of what its structure might be, FR or otherwise.

There is also only one event that reaches the opposite $Q_{s}=5$ extreme, and that is event \#24, shown explicitly in Figure 3. One of the features we look for in searching for evidence of an FR shape is a clear trailing edge for the FR, which is not always easy to discern. For event \#24, there is a bright prominence eruption, most clearly seen in the COR1 panels of Figure 3, which we believe can be interpreted as nicely outlining the bottom of an FR that is viewed face-on from the perspectives of STEREO-A and -B. Even south of the prominence, which fades quickly as the CME moves outwards, the HI1-B image in Figure 3 shows a front that can be interpreted as the trailing edge of the FR channel. Another crucial characteristic that we look for in assessing evidence for an FR shape is a clear dominant axis to the structure, indicating the orientation of the FR. The COR1 and COR2 images in Figure 3 make it very clear that this $\mathrm{CME}$ has a wide angular extent in the north-south direction. But from the frontal perspective provided by the LASCO/C3 image in Figure 3, it is clear that the CME is not very broad in the east-west direction. This CME has a dominant north-south orientation, consistent with an FR shape oriented roughly north-south. The 2008 April 26 event studied by Wood \& Howard (2009) would be another $Q_{s}=5$ event, with a circular appearance from STEREO-A's perspective suggesting an FR structure viewed edge-on, consistent with the clearly dominant east-west axis of the CME apparent from STEREO-B's frontal perspective.

Nearly half of our events are assigned the intermediate $Q_{s}=3$ value, which corresponds to an event that we believe we can model reasonably well with an FR shape, but which we suspect could also be modeled equally well with some other paradigm (e.g., the traditional "cone model"; Zhao et al. 2002). There is of course significant subjectivity involved in our $Q_{s}$ index, but with only 7 of our 28 events having $Q_{s}=4$ or 5 , we can use it to state that even in the STEREO era only about a quarter of CMEs in our estimation present an appearance in images that provides clear positive evidence for an FR morphology, preferable to other morphologies that might be assumed. This could be used as an argument against the FR paradigm being correct for all CMEs, but the overall case for the FR paradigm outlined in Section 1 still seems strong to us. Therefore, we instead believe that our difficulty in finding clear evidence for FR structures in all CMEs, even with extensive STEREO and SOHO imaging constraints, is indicative of the difficulties inherent in discerning magnetic structure from mass distributions, which will not necessarily outline the underlying magnetic structures very well.

\section{CHARACTERIZING FLUX ROPE PROPERTIES}

Many of the measured CME and FR characteristics from Tables 1 and 2 are displayed in the various panels of Figure 7. As in Figure 4(b) and Figure 5(a), we find it instructive to divide our sample of events into three groups according to associated surface activity, with group 1 being flare-associated events, group 2 being associated with filament eruptions (but no flares), and group 3 associated with no clear surface activity. Also analogous to those figures, we include the five additional events from Wood et al. (2016b), which are geoeffective events not perceived as MCs at Earth.

Figure 7(a) illustrates how the character of our CMEs changes significantly during the 2008-2012 period, indicating the shift from solar minimum towards solar maximum. The six 2009 events are all slow group 3 CMEs. In contrast, all but four of the events from 2011-2012 are fast group 1 events. Figure 7(b) shows a clear correlation between CME speed and flare strength, although there is still significant scatter.

The CME latitude and FR tilt angle are compared in Figure 7(c). Curiously, 28 of the 33 events have negative tilt angles (i.e., $\gamma_{s}<0^{\circ}$ ). In other words, the FRs tend to be tilted in a northeast-southwest direction as opposed to northwest-southeast. We can think of no systematic error that would produce this, nor can we think of any physical reason why there would be such a tendency. Thus, if not simply a statistical fluke, the cause of this bias remains a mystery. In any case, one purpose of Figure $7(\mathrm{c})$ is to look for a correlation between latitude and FR orientation. Due to differential rotation, filament channels in the northern hemisphere tend to have negative tilt angles, and those in the southern hemisphere tend to have positive tilts (Rust \& Kumar 1994). Thus, one might expect to see an 

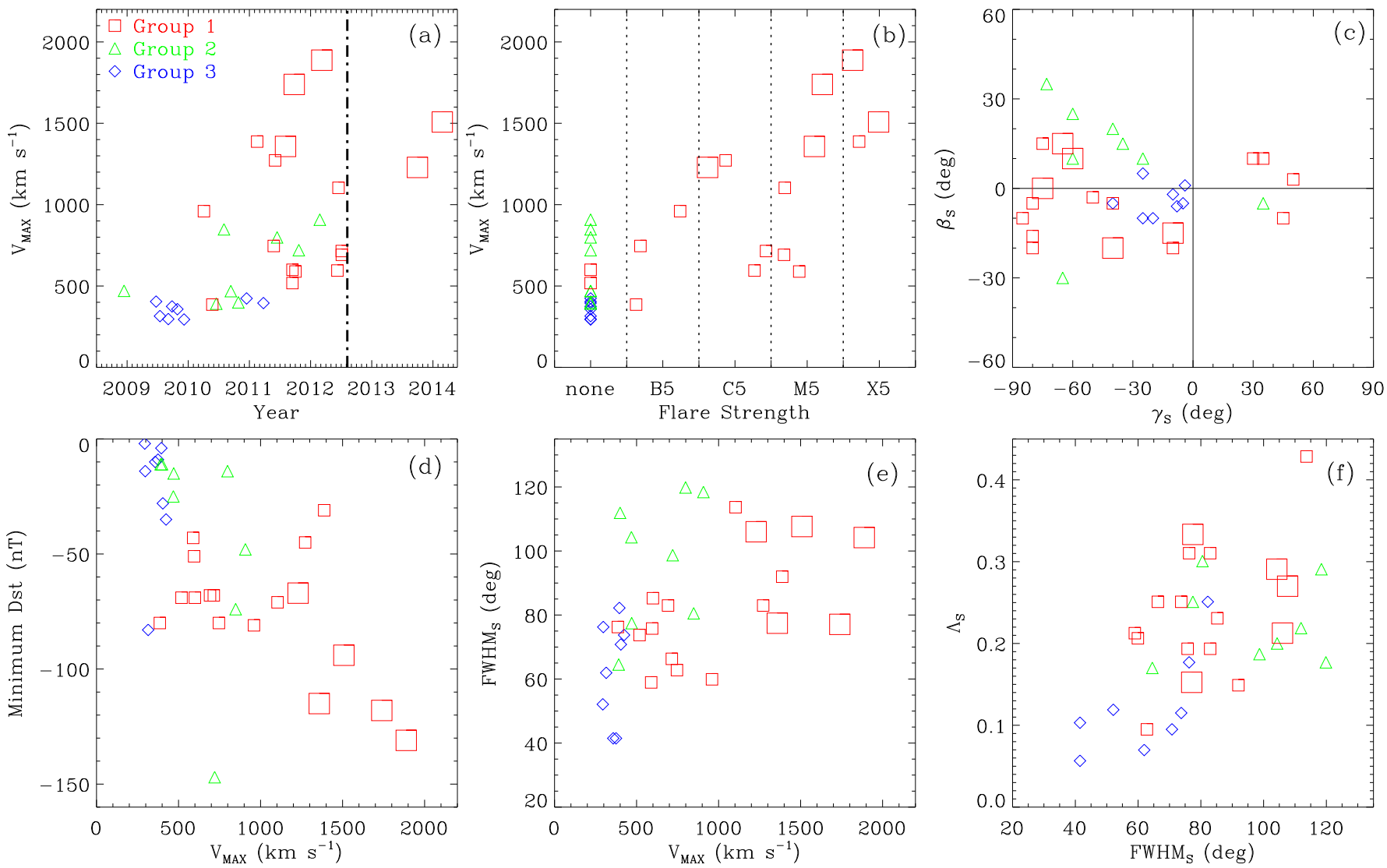

Figure 7. Plots comparing various characteristics of the CMEs and their fitted FR parameters, with the CMEs divided into Group 1 (flare-associated CMEs), Group 2 (filament eruption associated CMEs), and Group 3 (no surface activity). The five large boxes are for the five added events from Wood et al. (2016b). (a) Peak CME velocity plotted versus time. The dot-dashed line indicates the end of the MC survey period. (b) Peak velocity versus flare strength. (c) Latitude of CME trajectory versus FR tilt angle. (d) Minimum $D_{s t}$ value versus peak velocity. (e) FR full-width-at-half-maximum versus peak velocity. (f) FR aspect ratio (the "fatness" of the FR) versus FR width.

overabundance of events in the upper left and lower right quadrants of Figure $7(\mathrm{c})$. This is definitely not the case for the group 1 and group 3 events, but may be the case for group 2 events, all but one of which are in the expected quadrants, the exception being event \#13. It is the filament eruption associated group 2 events that would indeed most naturally be expected to show this behavior.

Figure 7(d) indicates some correlation between CME speed and geoeffectiveness as measured by $D_{s t}$. The minimum $D_{s t}$ ("disturbance storm time") listed in Table 2 measures the depression of the horizontal component of the geomagnetic field from a network of monitors near the equator, with large negative values indicating a significant geomagnetic storm. There is a great deal of scatter in Figure 7(d), and no correlation at all would be apparent without the addition of the 5 extra geoeffective events. Event \#23 looks like an outlier in this figure, with the most negative $D_{s t}$ in the figure despite a modest CME speed of only $V_{\max }=720.3 \mathrm{~km} \mathrm{~s}^{-1}$.

Figure 7(e) plots FR width versus $V_{\max }$. The three groups of CMEs are partly separated in this figure. The slow group 3 FRs are small, with $F W H M_{s}=63 \pm 16 \mathrm{deg}$. For group 1, there is a clear correlation between FR size and CME velocity. For $V_{\max }<1000 \mathrm{~km} \mathrm{~s}^{-1}$, the mean width and standard deviation are $F W H M_{s}=71 \pm 10$ deg; while for $V_{\max }>1000 \mathrm{~km} \mathrm{~s}^{-1}, F W H M_{s}=95 \pm 15 \mathrm{deg}$. The group $2 \mathrm{FRs}$ show a clear tendency to be larger than group 1 and 2 FRs with similar velocities, with $F W H M_{s}=97 \pm 21$ deg. It is two group 2 events (\#19 and \#24; see Appendix) that are in fact the largest FRs in our entire sample, suggesting that the largest CME FRs are associated with filament eruptions from large filament channels rather than X-ray bright active regions. It is only the shocks that make the fast $V_{\max }>1000 \mathrm{~km} \mathrm{~s}^{-1} \mathrm{CMEs}$ appear to be the largest of all CMEs. Our analysis suggests that their FR ejecta are not larger than the slower group 2 FRs. Figure $7(\mathrm{f})$ shows the FR aspect ratio as a function of FR width. There is a correlation, with the larger FRs being fatter, but it is not a tight correlation.

We find no correlation between the ellipticity of the FRs $\left(\eta_{s}\right)$ and $V_{\max }$, a correlation that one might expect if plowing 

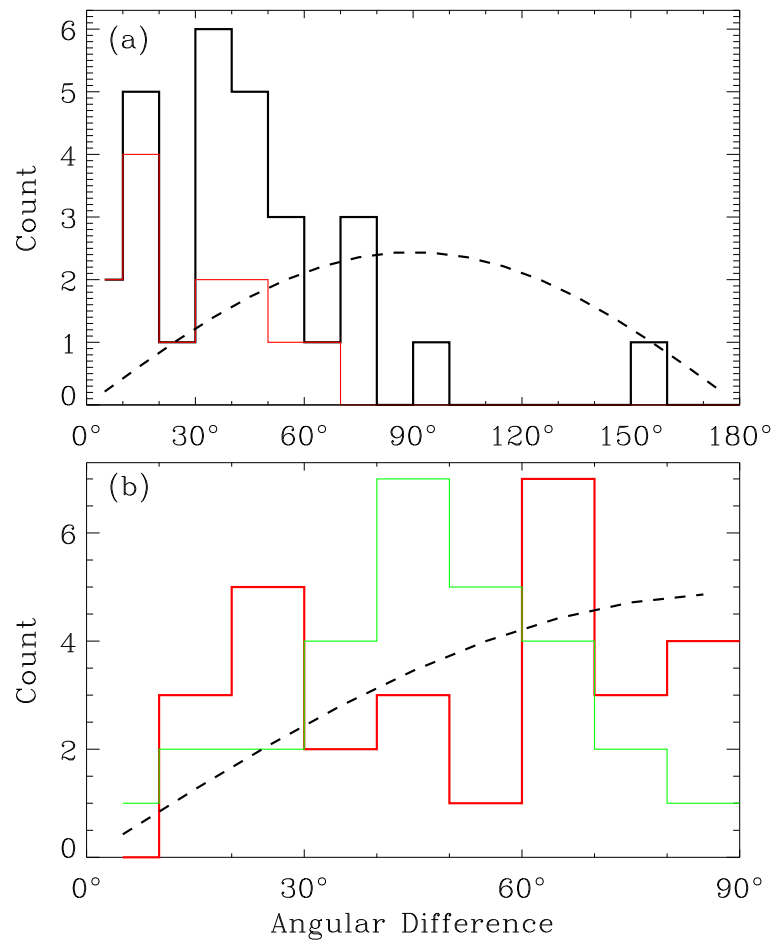

Figure 8. (a) Distributions of angular discrepancies between the two independent measurements of $\mathrm{MC}$ axis direction listed in Table $1,\left(\phi_{l}, \theta_{l}\right)$ and $\left(\phi_{n}, \theta_{n}\right)$, with the black line showing the full sample, and the red line excluding the poor $Q_{l}=3$ MCs. The dashed line is the distribution expected for no correlation between the two measurements. (b) Distributions of angular discrepancies between the image-based FR axis direction from Table $2\left(\phi_{s}, \theta_{s}\right)$ and the two MC axis direction measurements from Table 1, with the red line comparing with $\left(\phi_{l}, \theta_{l}\right)$ and the green line with $\left(\phi_{n}, \theta_{n}\right)$. The dashed line is the distribution expected if there is no agreement, and the two computed distributions agree relatively well with this, suggesting inconsistency between the MC orientations and the FR orientations inferred from imaging.

through a slower ambient solar wind yielded flattened FR channels. In particular, for fast events with $V_{\max }>1000$ $\mathrm{km} \mathrm{s}^{-1}, \eta_{s}=1.45 \pm 0.31$; little different from the value for slow events with $V_{\max }<1000 \mathrm{~km} \mathrm{~s}^{-1}, \eta_{s}=1.42 \pm 0.32$. The very modest ellipticity that we infer for our events is not in good agreement with past suggestions of substantial "pancaking" of the FR during interplanetary travel, corresponding to $\eta_{s} \approx 4-6$ or more (Riley \& Crooker 2004; Manchester et al. 2004b; Savani et al. 2011). Our measurements would be in better agreement with analyses implying or at least allowing for more modest flattening (Owens et al. 2006; Savani et al. 2010).

Finally, we can compare our CME trajectory directions in Table $2\left(\lambda_{s}, \beta_{s}\right)$ with the locations of associated surface activity, listed in Table $1\left(\lambda_{p}, \beta_{p}\right)$ The angular discrepancy between the two has a mean and standard deviation of $19.3 \pm 9.6 \mathrm{deg}$. Flares are much more compact than filament eruptions, so group 1 events have a better defined source location than group 2 events. Considering group 1 alone, we find a slightly lower angular discrepancy of $16.2 \pm 8.1 \mathrm{deg}$. These angular differences are generally interpreted as being due to actual deflections in the CME's trajectory early in its journey from the low corona (Kay et al. 2013, 2015).

\section{COMPARING IMAGING AND IN SITU PERCEPTIONS OF CME STRUCTURES}

One of the main reasons to use in situ Wind MC detections to define our STEREO CME sample was to allow comparison of FR characteristics inferred from imaging with $\mathrm{MC}$ characteristics inferred from the Wind data. Below we discuss three different aspects of this comparison: orientation, size, and velocity/expansion.

\subsection{Orientation}

As discussed in Section 2, Table 1 provides two independent assessments of MC orientation, both assuming an infinite cylinder geometry with the cylinder axes pointing towards $\left(\phi_{l}, \theta_{l}\right)$ and $\left(\phi_{n}, \theta_{n}\right)$ for the two measurements, respectively. We compute the angular discrepancy between these two measurements, and Figure 8(a) shows the distribution of these discrepancies. Also shown is a line indicating what the distribution would look like in the absence of any correlation, showing the distribution of orientation differences expected for two randomly oriented cylinders. The agreement is certainly better than random, but the average difference is still a rather large $44^{\circ}$. However, over half of our MC 
events have been flagged as poor $Q_{l}=3 \mathrm{MC}$ events (see Table 1). Figure 8(a) shows the distribution when the $Q_{l}=3$ events are ignored. This significantly improves the agreement, with the average difference decreasing to $28^{\circ}$.

However, the main goal here is to compare these MC orientations with those of the FRs reconstructed from the images. This is not a trivial exercise, however, as the $\gamma_{s}$ tilt angle parameter only indicates the FR axis orientation at the FR apex, and not where Wind actually encounters the FR in our reconstruction. Figure 6 shows Earth's path through the reconstructed FRs. At each point within the FR, we can find the shortest line connecting Earth to the FR axis. The plane normal to the FR axis containing this line then defines the FR orientation at that point. By computing this orientation at all points along Earth's path through the FR, and then averaging these orientations, we estimate an FR orientation for Earth in the infinite cylinder approximation, appropriate for comparison with the MC orientations. These measurements $\left(\phi_{s}, \theta_{s}\right)$ are listed in the last two columns of Table 2. For the MC orientation, the magnetic field direction along the central MC axis defines the direction quoted for the cylinder axis. However, images provide no such preferential direction, so both axis directions are possible. The direction quoted in Table 2 is the one with $\phi_{s}<180^{\circ}$, but the other axis direction $\left(\phi_{s}+180^{\circ},-\theta_{s}\right)$ is also possible.

Figure 8(b) compares the FR orientations with both MC orientation measurements, and provides a line indicating what the distribution should be in the absence of any correlation. We have to compare with both possible FR axis directions and take the minimum to represent the discrepancy, meaning the worst possible agreement is $90^{\circ}$ instead of $180^{\circ}$. The two distributions are similar to the non-correlation line, and the mean differences with $\left(\phi_{l}, \theta_{l}\right)$ and $\left(\phi_{n}, \theta_{n}\right)$ are $52^{\circ}$ and $47^{\circ}$, respectively, only a little better than the $60^{\circ}$ value expected for no correlation. We sought to improve agreement by excluding $Q_{l}=3 \mathrm{MCs}$ and cases where the analysis is potentially confused by multiple CMEs or consecutive MCs (see Appendix). For the remaining 9 events, the mean differences with $\left(\phi_{l}, \theta_{l}\right)$ and $\left(\phi_{n}, \theta_{n}\right)$ are $54^{\circ}$ and $45^{\circ}$, respectively, showing no improvement over the full sample. Thus, we conclude that the image-based FR orientations are simply not in good agreement with the MC orientations.

Inferences of CME morphology from images and in situ data both carry substantial uncertainties that may be the cause of the poor agreement seen in Figure 8(b). Since images only show mass and not magnetic field, the imagebased analysis involves the assumption that mass is loaded into the presumed FR in such a way as to reveal its shape, but this may not always be the case. The in situ analyses are hampered by the fundamental difficulty of inferring 3-D structure from a single, narrow, 1-D trace of plasma properties through that structure. There are also physical assumptions involved that can be overly simplistic (e.g. force-free, infinite cylinder, etc.). It is possible to change these assumptions, in particular to replace the cylindrical approximation with an elliptical or toroidal geometry (Hidalgo et al. 2002; Vandas \& Romashets 2003, 2015; Marubashi \& Lepping 2007; Hidalgo \& Nieves-Chinchilla 2012; Al-Haddad et al. 2013; Hu et al. 2014). It would be an interesting exercise to see if such assumptions would yield MC orientations in better agreement with the image-based inferences, but such an analysis is beyond the scope of this paper.

Although we do not in general claim to be certain at this point whether the image-based or MC-based orientation measurements are preferable, for the subset of events from 2009 we would claim that the image-based orientations are more plausible. The 2009 events are all slow events with no clear surface activity associated with them. They are all streamer blowout type CMEs that develop under the existing quiescent streamer structure, and therefore would be expected to have their orientation determined by that of the overlying streamer belt. All six of the 2009 CMEs are centered near the ecliptic plane, and inspection of source surface synoptic charts computed from magnetograms shows that the heliospheric current sheet was relatively flat and near the ecliptic plane at the locations of all six events. Thus, the expectation is that all six FRs should have a roughly east-west orientation, meaning we should see $\left|\gamma_{s}\right| \approx 0^{\circ}$, $\left|\theta_{l}\right| \approx 0^{\circ}$, and $\left|\theta_{n}\right| \approx 0^{\circ}$. The actual measurements suggest averages and standard deviations of $\left|\gamma_{s}\right|=14.5 \pm 13.7 \mathrm{deg}$, $\left|\theta_{l}\right|=43.3 \pm 15.7 \mathrm{deg}$, and $\left|\theta_{n}\right|=22.5 \pm 22.8 \mathrm{deg}$. The image-based $\gamma_{s}$ values best match the $0^{\circ}$ expectation, followed by $\theta_{n}$, and lastly by $\theta_{l}$. The fact that the $\left(\phi_{n}, \theta_{n}\right)$ measurements are noticeably more east-west than $\left(\phi_{l}, \theta_{l}\right)$ could be evidence that the prescription for relaxing the force-free assumption by Nieves-Chinchilla et al. (2016) leads to demonstrably more accurate MC analyses for these events.

\subsection{Size}

Our image-based reconstruction of the FR morphology and kinematics of the CME provides a prediction for how long Wind should encounter the FR, and these FR encounter times can be compared with the durations of the MCs observed by Wind. This comparison is made in Figure 1, which identifies the MC regions and also shows for each event the density peaks predicted by the image-based reconstruction, marking the predicted entry into and exit from the FR. (We are ignoring the shock density peaks for purposes of this discussion.) There are usually two separate peaks for the entry and exit, except for grazing incidence cases that show only one peak (e.g., event \#18). In some cases, 


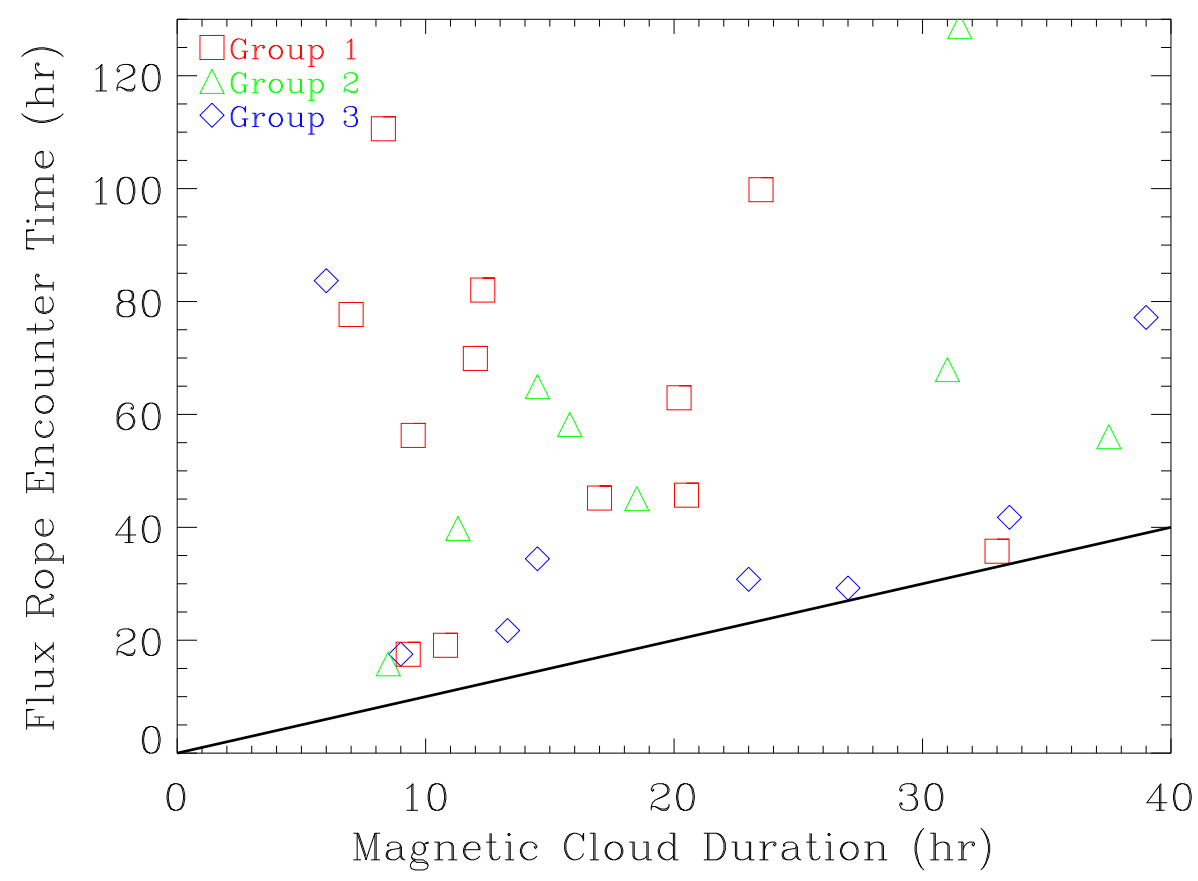

Figure 9. Flux rope encounter time at Wind predicted by the CME reconstruction from imaging data, plotted versus magnetic cloud duration observed at Wind, with the solid line being the line of agreement. Different symbols are used for Group 1 (flare-associated CMEs), Group 2 (filament eruption associated CMEs), and Group 3 (no surface activity) events.

the exit density peak is not visible in Figure 1 (e.g., event \#9) because it ends up beyond the the 3.5 day period used in the figure. These are typically cases where the Earth and Wind travel into the leg of the FR, resulting in a very late predicted exit time.

It is very clear from cursory inspection of Figure 1 that the predicted FR encounter times are generally much longer than the MC durations. In order to make a more clear comparison, we compute FR encounter times from the density peaks. For purposes of this calculation, we define the FR encounter time as follows. For each event we define a somewhat arbitrary density threshold of $15 \%$ of the maximum density, and we define the entry time as being when this threshold is first reached, and the exit time as when this density is last encountered.

The resulting FR encounter times are compared with the MC durations in Figure 9, where we once again divide the events into the three groups used in previous figures. It should be emphasized that some of these FR encounter times are surely unreasonably long. In the self-similar expansion approximation used in the FR reconstruction, the velocity at a point within the FR is proportional to its distance from the Sun. Thus, if Wind is predicted to exit from the leg of an FR at a third of the distance to the FR's leading edge, the velocity at that point will only be a third of the leading edge velocity, which is likely to be unreasonably slow in most cases, leading to an implausibly long FR encounter time. In reality, the ambient solar wind will be impinging on the trailing parts of the FR, presumably accelerating them to higher velocities than our reconstructions would suggest.

With this caveat in mind, Figure 9 illustrates the degree to which the FR encounter times are consistently longer than the MCs. The average MC duration is $18.5 \mathrm{hr}$, compared with $54.8 \mathrm{hr}$ for the average FR encounter time. The FRs inferred from imaging are simply much fatter than would be expected from the MC durations. Not surprisingly, the disagreement is less acute for the group 3 events, which are the narrowest FRs, having the lowest $\Lambda_{s}$ values. The substantial FR/MC size discrepancy suggests that there is not a clear one-to-one correspondence between the FR channels inferred from imaging and those seen as MCs by in situ instruments. Perhaps the true magnetic flux ropes are the narrow structures seen as MCs, but these FRs exist within larger ICME channels outlined by messier, more irregular magnetic barriers, representing the channels perceived as FR shapes in images. This could also explain the orientation differences between MCs and image-based FRs, as the MC orientation would not necessarily be identical to that of the larger ICME channel. 

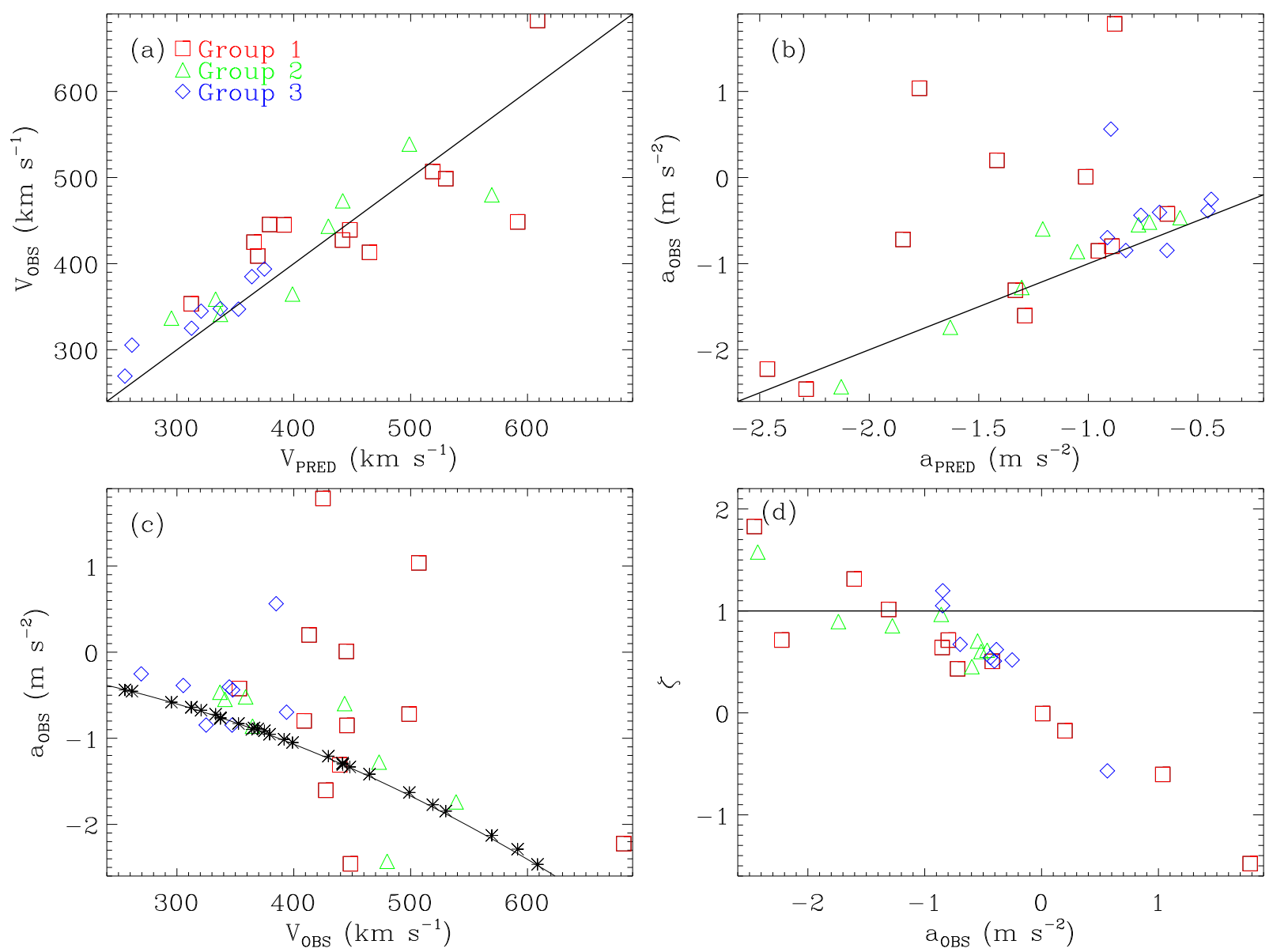

Figure 10. (a) Observed average velocity within the MC compared with that predicted by the image-based CME reconstruction, with the solid line being the line of agreement. Different symbols are used for Group 1 (flare-associated CMEs), Group 2 (filament eruption associated CMEs), and Group 3 (no surface activity) events. (b) Observed average acceleration within the MC compared with that predicted by the image-based CME reconstruction, with the solid line being the line of agreement. (c) Acceleration within the MC plotted versus velocity. The asterisks show the predicted instead of observed values, which follow the expected $a_{o b s}=-V_{o b s}^{2} / d$ relation for self-similar expansion. (d) The dimensionless expansion factor $\zeta$ versus acceleration within the MC.

Figure 1 compares velocities observed by Wind with velocities predicted by the image-based reconstructions. But comparing the velocities requires some care. This is especially true with regards to shock and shock sheath regions. The images will be indicating the shock propagation speed while the speeds measured by Wind will be the velocities of material moving through the shock, and these are not necessarily the same things (see discussion of event \#24 in the Appendix). The MC regions identified for our events should be CME ejecta, however, where the predicted velocities should unambiguously be in agreement with the observed velocities. Therefore, we here compare the predicted and observed velocities within the MC regions.

We compute mean observed and predicted velocities within the MC bounds ( $V_{\text {obs }}$ and $V_{\text {pred }}$, respectively), and compare them in Figure 10(a). The observed velocities are in reasonably good agreement with those predicted by the image-based reconstruction, with an average discrepancy of only $\pm 37 \mathrm{~km} \mathrm{~s}^{-1}$. This provides encouraging support for the accuracy of the kinematic aspects of the 3-D reconstructions. However, there are some curious systematic discrepancies. In particular, the reconstructions clearly tend to underpredict velocities for the slower CMEs, perhaps due to perturbations of the MC resulting in incursions of higher velocity material into the MC region.

The predicted velocities in Figure 1 all have negative slopes. This is a consequence of the self-similar expansion assumption used in the FR reconstruction process. With this assumption, velocity within the structure is proportional to distance from the Sun. Thus, trailing parts of the CME are slower, so velocity decreases with time. Inspection of the MC regions in Figure 1 shows that most do in fact show observed velocity decreases, qualitatively consistent with expectations. We perform linear fits to the observed and predicted velocities within the MC bounds, with the slopes of the resulting lines indicating accelerations seen at Wind ( $a_{o b s}$ and $a_{\text {pred }}$, respectively). These accelerations 
are compared in Figure 10(b). There is reasonable agreement for most events, with $a_{\text {obs }}$ falling within $\pm 0.35 \mathrm{~m} \mathrm{~s}{ }^{-2}$ of the prediction for $75 \%$ of the events, but there are a number of outliers, particularly five events that actually have positive $a_{o b s}$, suggesting a contracting MC instead of an expanding one.

The nature of the MC expansion can be explored further by combining the $V_{o b s}$ and $a_{\text {obs }}$ measurements. These two quantities are compared in Figure 10(c). For self-similar expansion, velocity and acceleration are related by $a_{o b s}=-V_{o b s}^{2} / d$ (see below), where $d$ is the distance from the Sun, and Figure 10(c) demonstrates that the measured $a_{\text {pred }}$ and $V_{\text {pred }}$ points naturally fit this relation. Most of the observed data points fall above this line, suggesting the observed MCs are not generally expanding as fast as the self-similar expansion approximation would predict. This is better explored using the dimensionless expansion parameter

$$
\zeta=-\frac{a_{o b s} d}{V_{o b s}^{2}},
$$

defined by Démoulin et al. (2008) and Démoulin \& Dasso (2009). For self-similar expansion, $\zeta=1$. Values of $\zeta>1$ indicate expansion faster than self-similar, $\zeta=0-1$ indicates expansion slower than self-similar, and $\zeta<0$ indicates contraction. Note that $\zeta$ is essentially identical to the $S_{r}$ self-similarity factor derived by Wood et al. (2016a) in the context of image analysis.

Figure 10(d) plots $\zeta$ versus $a_{o b s}$. Exactly $75 \%$ of the points fall in the range $\zeta=0.5-1.5$, which could be described as crudely self-similar. However, most of these points lie below $\zeta=1$. This is consistent with the measurements of Démoulin et al. (2008). Gulisano et al. (2010, 2012) find that MCs with relatively linear velocity trends, which they refer to as "non-perturbed" MCs, tend to have $\zeta \approx 1$. It is clear that many of our MCs would be considered "perturbed" in this regard, potentially accounting for the $\zeta<1$ tendency.

\section{PREDICTING 1 AU ARRIVAL TIMES}

The kinematic profiles in Figure 4(b) represent a significant improvement in our understanding of how CMEs propagate from the Sun to 1 AU, being constrained both by the observed Earth arrival time, and extensive STEREO monitoring continuously from the Sun to 1 AU. Since we know the ICME arrival times at Wind for all 33 of these events, these measurements can be used to explore how CME velocity at a given distance from the Sun relates to remaining travel time to Earth. This CME arrival time forecasting is useful for operational space weather forecasting purposes. Thus, we here explore the implications of our measurements for Earth arrival time predictions.

Figure 11(a) shows how the total Sun-to-Earth travel time correlates with peak CME speed. The slowest events in our sample, which reach only $V_{\max } \approx 300 \mathrm{~km} \mathrm{~s}^{-1}$, take $6-7$ days to make it to Earth. The fastest events with $V_{\max }>1000 \mathrm{~km} \mathrm{~s}^{-1}$ take only about $2-3$ days. However, for forecasting purposes, it is not peak CME speed that one wants to relate to $1 \mathrm{AU}$ travel time, but CME speed at a particular height. We can use the kinematic models in Figure 4(b) to tell us the leading edge speed of all of the CMEs at any distance $D$, and knowing the ICME arrival times at Earth, we can compare these speeds with the remaining travel time from distance $D$ to Earth. This is done in Figure 11(b) for $D=0.025,0.3$, and 0.6 AU. We fit these curves with third order polynomials, such that the 1 AU travel time (in days) is

$$
t_{A U}=\sum_{i=0}^{3} a_{i} V^{i},
$$

where $V$ is the velocity at distance $D$ in $\mathrm{km} \mathrm{s}^{-1}$. The $a_{i}$ coefficients are listed in Table 3 for a range of $D$ values between 0.025 and 0.6 AU, allowing travel times to Earth to be predicted from a measured CME velocity anywhere in this distance range. This prescription will only apply within the velocity range covered by our sample of events, which is given by the $\left(V_{-}, V_{+}\right)$values listed in Table 3 .

The scatter about the polynomial fits in Figure 11(b) can be used to estimate uncertainties in the $t_{A U}$ predictions. The most natural estimate is the standard deviation of the discrepancies from the fit, $\sigma_{t}$, which is listed in the last column of Table 3. But in the literature, sometimes the mean discrepancy, $\overline{\delta t_{A U}}$, is provided instead, so we also list this value in the table. Figure $11(\mathrm{c})$ plots both $\sigma_{t}$ and $\overline{\delta t_{A U}}$ versus $D$. Close to the Sun at $D=0.025$ AU the travel time uncertainty is $\sigma_{t}=11.7 \mathrm{hr}$. This value quickly drops as $D$ increases, reaching a minimum at about $D=0.3 \mathrm{AU}$, where $\sigma_{t}=6.3 \mathrm{hr}$ (and $\overline{\delta t_{A U}}=4.6 \mathrm{hr}$ ). The reason for the minimum at $D=0.3 \mathrm{AU}$ is that by this distance most CMEs have finally reached their final, terminal speeds, with velocities that do not change much after this (see Figures 4 (b) and $5(\mathrm{~b}))$. The uncertainties do not decrease any further with increasing $D$.

The velocities shown in Figure 4(b) and used in the analysis depicted in Figures 11(a-c) are leading edge velocities. One potentially significant contributor to the uncertainties $\sigma_{t}$ and $\overline{\delta t_{A U}}$ is that Earth is not always hit near the leading 

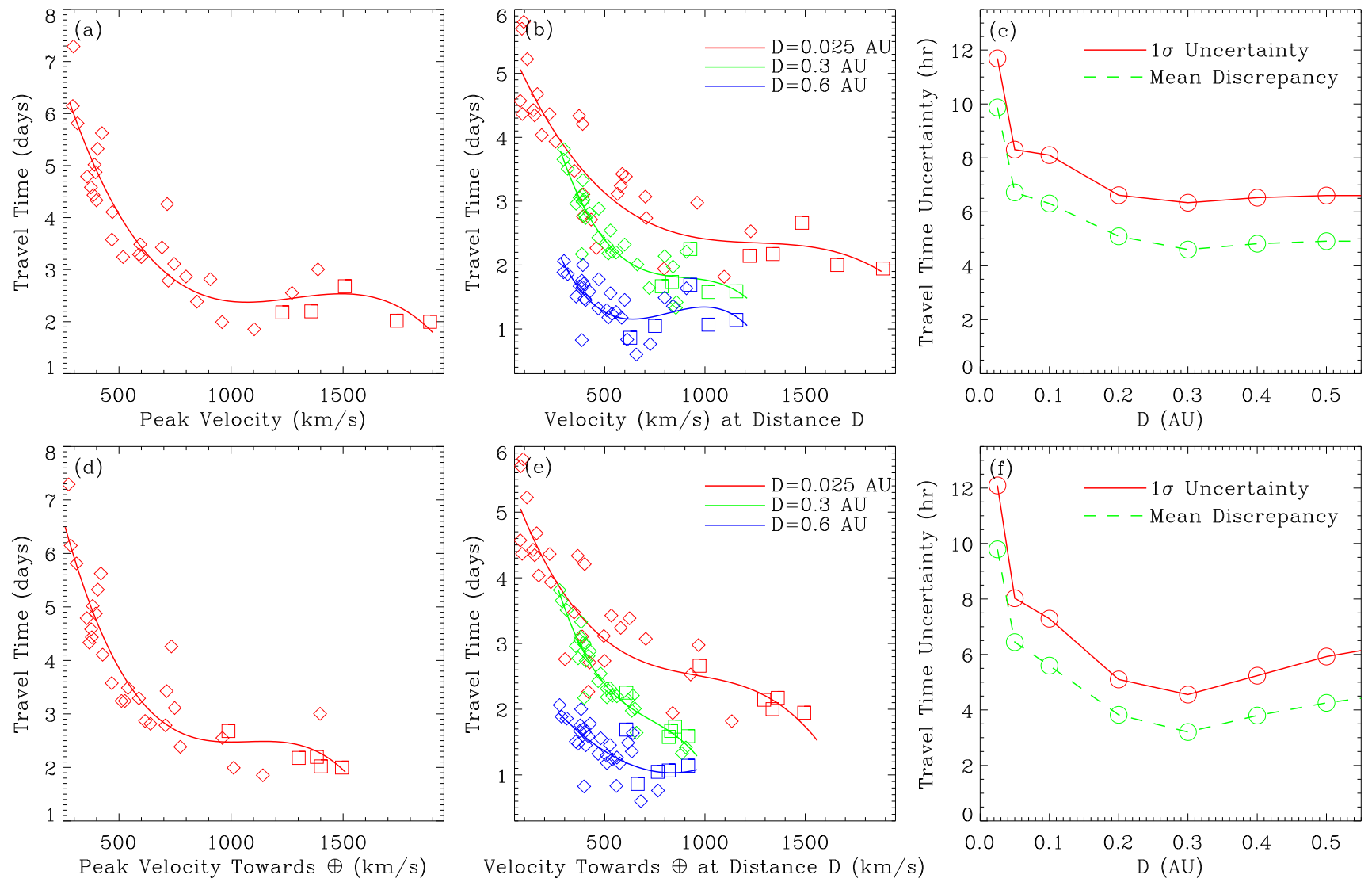

Figure 11. (a) Travel time from the Sun to Earth as a function of peak CME velocity at its leading edge. Diamonds are the MC sample of events from Table 1, and boxes are the five included events from Wood et al. (2016b). A third-order polynomial is fitted to the data points. (b) Travel time from distance D to Earth as a function of CME leading edge velocity measured at distance $\mathrm{D}$, shown for 3 values of $\mathrm{D}$. Third-order polynomials are fitted to the curves, with the fit coefficients listed in Table 3 . (c) Uncertainties in travel time estimates to Earth as a function of distance where a CME's leading edge velocity is measured, computed from the scatter about curves like those in (b). Panels (d)-(f) are analogous to panels (a)-(c), but instead of leading edge velocity, the velocity used is the velocity of the part of the CME moving towards Earth.

edge of a CME. In many cases Earth is hit by a part of the CME front well behind the leading edge, and therefore traveling slower. Because our analysis involves a full morphological reconstruction of the CMEs, we know what the actual CME speeds towards Earth are, and we can make a version of Figure 4(b) with those velocities instead of the leading edge velocities. Figure 11(d-f) repeats the analysis of Figure 11(a-c), but using these Earth-directed CME speeds, in order to see if this significantly reduces uncertainties. The results, which are also listed in Table 3, are unexpected. There is significant decrease in $\sigma_{t}$ and $\overline{\delta t_{A U}}$ only at $D=0.2-0.4 \mathrm{AU}$. At $D=0.3 \mathrm{AU}$, the uncertainties dip to an impressively low $\sigma_{t}=4.6 \mathrm{hr}$ (and $\overline{\delta t_{A U}}=3.2 \mathrm{hr}$ ), but they then actually increase for $D>0.3 \mathrm{AU}$, which is counterintuitive.

In order to explain this, we note that there are two primary sources of uncertainty: (A) the uncertainty in the velocity measurements providing the basis for this calculation (i.e., Figure 4(b)); and (B) the intrinsic variation in $1 \mathrm{AU}$ travel time for a set of CMEs at a given velocity at a given distance $\mathrm{D}$, which may have different 1 AU travel times despite the same starting conditions, due to different solar wind conditions between distance D and Earth. The increase in uncertainty for $D>0.3 \mathrm{AU}$ seen in Figure 11(f) can really only be explained by option (A). It is quite possible that our velocity measurements are more uncertain for $D>0.3 \mathrm{AU}$. This may be because measurements from the HI2 field of view are simply intrinsically more uncertain, or it could be an artifact of our particular method of velocity measurement, assuming a few phases of constant velocity or acceleration, and always assuming a final, relatively lengthy phase of constant velocity (see Section 3.1).

In practice, accurately estimating a Sun-Earth line CME velocity in a real time forecasting situation will not be easy, unless one actually has a spacecraft operating much closer to the Sun on the Sun-Earth line. For now, the limited improvement in $\sigma_{t}$ and $\overline{\delta t_{A U}}$ is probably not sufficient to be worth the extra effort. We suspect that using the leading 
Table 3. Relating CME Velocity to 1 AU Travel Time

\begin{tabular}{|c|c|c|c|c|c|c|c|c|}
\hline \multirow[b]{2}{*}{$\begin{array}{c}D^{a} \\
(\mathrm{AU})\end{array}$} & \multirow[b]{2}{*}{$\begin{array}{c}V_{-}^{b} \\
\left(\mathrm{~km} \mathrm{~s}^{-1}\right)\end{array}$} & \multirow[b]{2}{*}{$\begin{array}{c}V_{+}{ }^{b} \\
\left(\mathrm{~km} \mathrm{~s}^{-1}\right)\end{array}$} & \multicolumn{4}{|c|}{ Coefficients $^{c}$} & \multirow[b]{2}{*}{$\begin{array}{c}{\overline{\delta t_{A U}}}{ }^{(\mathrm{hr})}\end{array}$} & \multirow[b]{2}{*}{$\begin{array}{l}\sigma_{t}{ }^{e} \\
(\mathrm{hr})\end{array}$} \\
\hline & & & $a_{0}$ & $\begin{array}{c}a_{1} \\
\left(10^{-3}\right) \\
\end{array}$ & $\begin{array}{c}a_{2} \\
\left(10^{-6}\right) \\
\end{array}$ & $\begin{array}{c}a_{3} \\
\left(10^{-9}\right) \\
\end{array}$ & & \\
\hline \multicolumn{9}{|c|}{ Analysis with Leading Edge Velocities } \\
\hline 0.025 & 78.7 & 1887.7 & 5.63 & -7.55 & 5.88 & -1.55 & 9.9 & 11.7 \\
\hline 0.05 & 141.5 & 1861.1 & 6.00 & -7.64 & 5.05 & -1.13 & 6.7 & 8.3 \\
\hline 0.10 & 203.9 & 1593.8 & 6.51 & -9.53 & 6.69 & -1.55 & 6.3 & 8.1 \\
\hline 0.20 & 291.0 & 1156.6 & 7.90 & -17.4 & 17.1 & -5.67 & 5.1 & 6.6 \\
\hline 0.30 & 294.2 & 1156.6 & 7.59 & -18.8 & 20.6 & -7.67 & 4.6 & 6.3 \\
\hline 0.40 & 294.2 & 1156.6 & 7.05 & -19.1 & 22.2 & -8.54 & 4.8 & 6.5 \\
\hline 0.50 & 294.2 & 1156.6 & 6.29 & -18.4 & 22.7 & -9.07 & 4.9 & 6.6 \\
\hline 0.60 & 294.2 & 1156.6 & 5.18 & -16.0 & 20.6 & -8.38 & 4.9 & 6.6 \\
\hline \multicolumn{9}{|c|}{ Analysis with Velocities Towards Earth } \\
\hline 0.025 & 79.0 & 1494.7 & 5.71 & -8.87 & 8.68 & -3.02 & 9.8 & 12.1 \\
\hline 0.05 & 136.8 & 1473.7 & 6.00 & -7.78 & 4.99 & -1.05 & 6.4 & 8.0 \\
\hline 0.10 & 197.2 & 1262.0 & 6.38 & -8.67 & 4.59 & -0.38 & 5.6 & 7.3 \\
\hline 0.20 & 275.0 & 1065.9 & 7.66 & -16.3 & 15.2 & -4.86 & 3.8 & 5.1 \\
\hline 0.30 & 275.0 & 915.8 & 7.80 & -21.1 & 26.5 & -12.1 & 3.2 & 4.6 \\
\hline 0.40 & 275.0 & 915.8 & 5.67 & -12.4 & 12.6 & -4.74 & 3.8 & 5.2 \\
\hline 0.50 & 275.0 & 915.8 & 4.37 & -8.60 & 7.58 & -2.18 & 4.3 & 5.9 \\
\hline 0.60 & 275.0 & 915.8 & 2.96 & -4.37 & 2.18 & 0.35 & 4.6 & 6.4 \\
\hline
\end{tabular}

Note - ${ }^{a}$ Distance where velocity measurement made. ${ }^{b}\left(V_{-}, V_{+}\right)$is the allowable range of velocities. ${ }^{c}$ Coefficients of the third order polynomial fit to compute the 1 AU travel time (see equation (6)). ${ }^{d}$ Mean discrepancy with the Earth arrival time prediction. ${ }^{e}$ Standard deviation of discrepancies with the Earth arrival time prediction.

edge velocities is more practical. Though even here we should emphasize that an effort must be made to estimate the true 3-D leading edge velocity of the CME, and not just a projected velocity in an image plane, which would not be appropriate to use in equation (6).

In principle, the arrival time uncertainties of our proposed forecasting prescription can be compared with other published assessments (Gopalswamy et al. 2000, 2001; Kilpua et al. 2012; Millward et al. 2013; Möstl et al. 2014; Vršnak et al. 2014; Mays et al. 2015; Rollett et al. 2016), but in practice this is not trivial, as different analyses assume different constraints available at different distances from the Sun, and use samples of CMEs with different distributions of fast and slow events. Some quoted $1 \mathrm{AU}$ travel time uncertainties are for predictions for the full travel time from near the Sun to $1 \mathrm{AU}$, and some are for predictions after the CME is already within a day or so of reaching Earth. The last two columns of Table 3 by themselves represent many different quantifications for 1 AU travel time forecasting accuracy. We believe the uncertainties quoted for our prescription in Table 3 are competitively low compared to ones cited in the papers cited above. But it should be emphasized that in an actual real time forecast, these uncertainties would have to be added in quadrature with the errors introduced by the uncertainty in the velocity measured for that particular event, and it is that uncertainty that may end up dominating the accuracy of the $1 \mathrm{AU}$ travel time estimate.

\section{SUMMARY}

We have conducted a survey of MC CMEs observed by Wind from 2008 to mid-2012 that can be tracked from the Sun to near 1 AU by STEREO, performing a 3-D reconstruction of each event's morphology and kinematic behavior based on STEREO and SOHO/LASCO imagery. Our principle results are summarized as follows:

1. Out of 48 Wind MCs, 31 are definitively connected with CMEs trackable to near 1 AU by STEREO. Most of the remaining 17 unaffiliated MCs are probably CMEs as well, but they may be too faint or confused with other $\mathrm{CME} / \mathrm{CIR}$ fronts in the images for a definitive connection.

2. Our 3-D CME reconstruction approach assumes an FR morphology. We find the FR paradigm to be reasonably 
successful at reproducing CME appearance in the images, but in our judgment only about a quarter of the events provide sufficient evidence for an FR shape to suggest that the FR paradigm is clearly preferable to other paradigms for CME morphology (e.g., the cone model).

3. The FR orientations from our image-based reconstructions are not in good agreement with MC orientations inferred from Wind data, and the FRs are usually too large to be consistent with MC durations.

4. Focusing only on six streamer blowout CMEs from 2009, which should have roughly east-west FR orientations, the image-based reconstructions generally recover the expected orientations. The MC analyses of the Wind data are not as successful in this regard, but there seems to be significant improvement when the traditional force-free assumption is relaxed.

5. The image-based reconstructions reproduce the observed mean velocities within MC regions to within $\pm 37 \mathrm{~km} \mathrm{~s}^{-1}$ on average. The reconstructions assume self-similar expansion. Exactly $75 \%$ of the MCs exhibit accelerations crudely consistent with self-similar expansion $(\zeta=0.5-1.5)$, though there is a clear tendency for slower expansion rates $(\zeta<1)$, probably due to perturbations of the MCs.

6. Our reconstructions provide full Sun-to-Earth kinematic measurements. After supplementing our main sample with five geoeffective events from Wood et al. (2016b), we find that 14 of 16 CMEs with $V_{\max }>600 \mathrm{~km} \mathrm{~s}^{-1}$ have measurable deceleration in the interplanetary medium after reaching their peak velocity.

7. We use the kinematic models to provide simple prescriptions for predicting CME arrival times at Earth. These prescriptions are provided for a range of distances from the Sun where CME velocity measurements might be made $\left(D=0.025-0.6 \mathrm{AU}\right.$ ). The $1 \sigma$ uncertainties in these predictions range from $\sigma_{t}=4.6-11.7 \mathrm{hr}$ (or mean discrepancies of $\overline{\delta t_{A U}}=3.2-9.9 \mathrm{hr}$ ), depending on the distance $D$ of the velocity measurement and on whether that velocity is a leading edge velocity or a velocity along the Sun-Earth line.

8. We find it instructive to group our events based on associated surface activity: group 1 (flare-associated CMEs), group 2 (filament eruption associated CMEs), and group 3 (no surface activity). These groups exhibit noticeably different kinematic behavior, with clear differences in peak velocities $\left(V_{\max }\right)$ and heights where $V_{\max }$ is first reached. For group $3, V_{\max }=294.2-423.8 \mathrm{~km} \mathrm{~s}^{-1}$ and $R=29.4 \pm 14.8 \mathrm{R}_{\odot}$; while for group $1, V_{\max }>500$ $\mathrm{km} \mathrm{s}^{-1}$ for all but one event, and $R=3.2 \pm 2.2 \mathrm{R}_{\odot}$. The group 2 events are intermediate, with $V_{\max }=391.0-907.6$ $\mathrm{km} \mathrm{s}^{-1}$ and $R=13.9 \pm 3.8 \mathrm{R}_{\odot}$.

9. The three groups are partially separated in parameter space when FR width is plotted versus $V_{\max }$, with group 3 events being slow and relatively narrow, group 1 events showing a correlation of width with $V_{\text {max }}$, and group 2 events being generally broader than the other events at similar speeds. Our two broadest modeled FRs are both group 2 events.

Financial support was provided by the Chief of Naval Research, and by NASA awards NNH10AN83I and NNH14AX61I to the Naval Research Laboratory. TNC is partially supported by the National Science Foundation under AGS-1433086 and the NASA/LWS program. The STEREO/SECCHI data are produced by a consortium of NRL (US), LMSAL (US), NASA/GSFC (US), RAL (UK), UBHAM (UK), MPS (Germany), CSL (Belgium), IOTA (France), and IAS (France). We acknowledge use of NASA/GSFC's Space Physics Data Facility's OMNIWeb (or CDAWeb or ftp) service, and OMNI data. The $D_{s t}$ values used in this paper are from Kyoto's World Data Center for Geomagnetism (http://wdc.kugi.kyoto-u.ac.jp/dstdir/index.html).

\section{APPENDIX}

\section{A. NOTES ON INDIVIDUAL EVENTS}

We here provide a brief discussion of the individual events, particularly important for cases in which multiple CMEs make interpretation of Figure 1 and Figure 6 difficult. Movies of each event showing the STEREO and SOHO/LASCO images of these CMEs, compared with synthetic images from the CME reconstructions described above, are provided in the online version of this article. 
Event \#1: This is a well-studied event that was the first clear case of an Earth-directed CME continuously trackable all the way from the Sun to 1 AU (Davis et al. 2009; Liu et al. 2010; DeForest et al. 2011). Our analysis has the FR hitting Earth near our estimated ICME arrival time, which is about 18 hours earlier than the MC start time (see Figure 1).

Event \#2: This is a CME that changes appearance significantly as it expands away from the Sun, meaning the self-similar expansion approximation is unusually poor in this case. We have already published a description of a more complex, time-dependent analysis of this event (Wood et al. 2012a), to which we refer the reader for details.

Event \#3: This is a faint, narrow CME from the perspective of both STEREO-A and -B, which is undetectable in the SOHO/LASCO images. The in situ ICME signature, shown in more detail by Kilpua et al. (2014), is probably the weakest of all our events, with only a very small density and field increase. A CIR arrives a day after our estimated ICME arrival time, producing far more dramatic density, velocity, and field increases.

Event \#4: This is a particularly instructive case for illustrating the value of multiple viewpoints for correct interpretion. As shown in Figure 6, our analysis reveals two separate CMEs, the first (CME1) beginning at about 15:00 on 2009 September 2, directed west of Earth, which hits STEREO-A on September 8. It is the second (CME2), which starts about 10 hours later, that hits Earth, though the center of the CME is well to the east of Earth. From SOHO/LASCO's perspective, CME1 is readily apparent, but CME2 is not clearly discernible as a separate eruption. From STEREO-A's perspective the situation is reversed - CME1 is nearly invisible, and what evidence there is of it is not easy to discern as being distinct from CME2, which is relatively bright. It is only STEREO-B that provides a perspective that makes it clear that there are two distinct slow streamer blowout CMEs. This may be a case where the first streamer blowout CME triggered an eruption from an adjacent part of the streamer belt, resulting in CME2, which ultimately yielded the MC at Earth. This event is also notable for having the longest $1 \mathrm{AU}$ travel time in our sample, taking over 7 days to get from the Sun to Earth.

Event \#5: We have already mentioned in Section 3.2 how this particularly faint, jet-like event is the only $Q_{s}=1$ CME in our sample, providing no evidence of an FR morphology, although the reconstructed FR nevertheless ends up matching the MC time range well in Figure 1.

Event \#6: This is another faint, streamer blowout CME from 2009, with only very weak signatures in SOHO/LASCO. In COR1, the CME reaches the edge of the COR1-B field of view well before it reaches the edge of COR1-A, but this changes by the time the CME exits COR2, suggesting the CME may have started out with a more westerly trajectory, quickly deflecting eastwards in the COR2 field of view. We cannot reproduce this in our reconstruction without abandoning the self-similar expansion approximation.

Event \#7: This CME seems to be part of a general outflow from part of the streamer belt, which makes it very difficult to decide what part of this outflow represents the leading edge of the CME. The Earth arrival time constraint provided by the Wind data is crucial for deciding what front to follow as the leading edge. Unlike many of the other 2009 streamer blowout CMEs, there is actually a nice SOHO/LASCO partial halo for this event, which is very helpful for constraining the FR orientation.

Event \#8: This is a very well studied event, notable for being the first truly geoeffective CME of solar cycle 24 (Möstl et al. 2010; Rouillard et al. 2011). Our reconstruction of this event has already been published (Wood et al. 2011), as well as being discussed at length in the context of our survey of geoeffective events (Wood et al. 2016b), so we do not discuss the reconstruction further here. There are two overlapping MCs associated with this event (see Figure 1), but there is clearly only one CME to account for both of them. The Earth arrival time of our model FR is in better agreement with the first MC, and its orientation is in better agreement with it as well, so we simply associate the FR with that MC in Table 1. But the FR encounter time suggested by the rather fat reconstructed FR is more than long enough to encompass both MCs.

Event \#9: There are two large, bright CMEs launched roughly towards Earth, and both are reconstructed (see Figure 6). The first one (CME1), launched at 16:35 on 2010 May 23, is the one we associate with the MC. The faster second one (CME2) erupts about 21 hours later. It decelerates to a similar final speed to CME1, so it never overtakes CME1. Figure 6 shows the situation as the CME1 FR is hitting Earth. Unlike CME1, CME2 has a very clear shock structure around it, which is modeled in our reconstruction as well. Our reconstruction 
has the CME2 shock hitting Earth, but the CME2 FR misses to the west, which is why we associate the MC with CME1. The CME1 arrival time shown in Figure 1 is about 4 hours after the ICME start time, but about 12 hours before the MC start time. Our reconstruction actually has the CME2 shock hitting Earth at about the MC start time, leading to the interesting question, what effect does the CME2 shock have on CME1, and is there really any evidence for the CME2 shock in the in situ data? We do not think so, and it seems to us likely that the CME2 shock seen clearly in the imaging does not actually extend inside the FR of CME1. This contradicts the reconstruction in Figure 6, but this is simply a limitation of the kind of reconstruction we are doing. In multi-event reconstructions of this nature we can only reconstruct events independently and then superimpose them, which can potentially be misleading. Lugaz et al. (2012a) provide a detailed assessment of the evidence for interaction between these two CMEs. Our CME1 trajectory and FR tilt angle from Table $5\left(\lambda_{s}=12^{\circ}, \beta_{s}=3^{\circ}\right.$, $\left.\gamma_{s}=50^{\circ}\right)$ can be compared with the $\left(\lambda_{s}=10^{\circ}, \beta_{s}=0^{\circ}, \gamma_{s}=65^{\circ}\right)$ measurement of Lugaz et al. (2012a). Finally, we note that with $V_{\max }=385.9 \mathrm{~km} \mathrm{~s}^{-1}$, CME1 is the slowest flare-associated CME in our sample, the only flare-associated event that never exceeds $500 \mathrm{~km} \mathrm{~s}^{-1}$.

Event \#10: This CME is notable for early rotation (Vourlidas et al. 2011), and for hitting the MESSENGER spacecraft in addition to hitting Earth (Nieves-Chinchilla et al. 2012). The time-dependent behavior noted by Vourlidas et al. (2011) means self-similar expansion is not a very good approximation close to the Sun. Thus, our reconstruction fits the COR1 data poorly, as we have focused more on reproducing the COR2 and HI1 appearance well. There is a CME that erupts about 10 hours after our event. We believe this CME misses Earth to the east, and in our judgment remains separated from our event to the extent that we do not do a reconstruction for it, but this second CME confuses interpretation of the SOHO/LASCO images somewhat.

Event \#11: This is an extensively analyzed event, popular because of its geoeffectiveness, and for studying CMECME interaction (Schrijver \& Title 2011; Wu et al. 2011; Temmer et al. 2012; Harrison et al. 2012; Möstl et al. 2012; Webb et al. 2013). However, from an imaging perspective, this is a frustrating event, because data gaps mean there is no available SOHO/LASCO data, and STEREO-B data gaps lead to minimal COR2-B and HI1-B constraints. For our reconstruction, we ultimately decide to focus on only two of the 5 or 6 events discussed in the literature cited above: CME2 and CME3 launched at about 8:00 and 8:30 on 2010 August 1, respectively. There is an earlier event (CME1) from about 3:10 that is also relevant for this discussion, which appears to be swept up and obliterated by the faster, brighter CME2, early in the HI1-A field of view. Figure 6 shows the situation as the shock of CME2 approaches Earth. It is the CME2 shock that is responsible for the shock that marks the ICME start time in Figure 1. Our reconstruction has the shock arriving about 5 hours too early. However, this reconstruction has the FR of CME2 missing Earth entirely, meaning we ultimately do not associate CME2 with either of the 2 consecutive MCs shown in Figure 1. Our reconstruction has the FR of CME3 reaching Wind near the start time of the second MC, so it is the second MC that we connect with CME3 in Table 1. The orientation of $\mathrm{MC \# 2}$ is also in better agreement with the $\mathrm{FR}$ orientation than is the case for $\mathrm{MC \# 1}$. It is possible that the short MC\#1 may be associated with the swept up remnants of CME1, as suggested by Möstl et al. (2012).

Event \#12: The FR encounter time implied by the reconstruction is much longer than the MC time, but there are density peaks relatively close to both the FR entry and exit times.

Event \#13: This event looks like a nice north-south oriented FR CME, somewhat analogous to event \#24 shown in Figure 3 . The predicted ICME arrival time at Wind nicely matches an observed density peak at that time (see Figure 1), but we have assumed an ICME arrival time about 14 hours earlier, where a velocity jump is observed along with a smaller density increase. Complicating interpretation further is that the MC start time is actually about 12 hours after the predicted ICME arrival. This illustrates the kinds of difficulties that can arise when trying to connect a front followed in images with features in in situ data.

Event \#14: This is a CME with a particularly slow, gradual initial expansion. This event and event \#2 are the only events in our sample that take over 50 hours to reach their peak speeds of $V_{\max } \approx 400 \mathrm{~km} \mathrm{~s}^{-1}$, by which time they are both well into the HI1 field of view.

Event \#15: This event has garnered significant attention for being associated with the first X-class flare of solar cycle 24, and for providing another interesting example of CME-CME interaction (Schrijver et al. 2011; Maričić et al. 2014; Mishra \& Srivastava 2014; Temmer et al. 2014). Our reconstruction involves two CMEs: a slow CME (CME1) that begins at about 16:24 on 2011 February 14, followed by a faster CME (CME2) at 1:55 
on 2011 February 15, which is the one associated with the X-flare. It is only CME2 that ultimately hits Earth in our reconstruction. It catches up with CME1 in the HI1 field of view, and seems to nudge it further to the north than it already was. With a peak speed of $V_{\max }=1387.8 \mathrm{~km} \mathrm{~s}^{-1}$, CME2 ends up being the fastest CME in our sample, and has a visible shock that is modeled in the reconstruction. The kinematic model for this event in Figure 4(b) is unusual for having two deceleration phases instead of just one.

Event \#16: This is the final slow, streamer blowout type CME in our sample, with no clear surface activity associated with it. A faint but complete halo is apparent in the SOHO/LASCO images. Self-similar expansion is not a terribly good approximation for this event, limiting the degree to which our model can reproduce the CME appearance in the images.

Event \#17: Our reconstruction involves two CMEs, the first (CME1) starting at 4:20 on 2011 May 25, and the second (CME2) starting at about 12:50. Both are quite faint in COR1 and in LASCO. The faster CME2 catches up to and slightly overtakes CME1 in the HI1 field of view. Figure 6 shows the situation as CME1 is reaching Earth. Our reconstruction has CME2 just barely missing Earth to the west, but this is a case where there is significant doubt as to which of the two CMEs is responsible for the MC. The big velocity increase after the MC period in Figure 1 suggests the CME may have arrived at Earth in the midst of a CIR.

Event \#18: This event was analyzed in the previous survey of cycle 24 geoeffective events (Wood et al. 2016b). Our reconstruction is described in detail in this previous work. There are three modeled CMEs in this difficult reconstruction (CME1, CME2, CME3), all emanating from the same active region, with the three CMEs seeming to merge in the HI1 field of view. There is some uncertainty regarding whether CME2 and/or CME3 are Earthdirected, but our reconstruction ultimately suggests that only CME3, the fastest and largest of the three CMEs, encounters Earth, and it only just barely grazes it. There are two consecutive MCs identified in the Wind data. We tentatively identify the CME with the longer second one, as the FR orientation seems more consistent with this one.

Event \#19: Our reconstruction has the Earth just barely grazing the bottom of the west leg of this CME. This event and event \#24 shown in Figure 3 have several interesting characteristics in common. Both are associated with filament eruptions from very large filament channels, but no flares. There is no impulsive acceleration, but both gradually accelerate to surprisingly high speeds of $V_{\max }=798.9 \mathrm{~km} \mathrm{~s}^{-1}$ and $V_{\max }=907.6 \mathrm{~km} \mathrm{~s}^{-1}$, respectively; and they do not decelerate, making these the only CMEs in our sample with $V_{\max }>600 \mathrm{~km} \mathrm{~s}^{-1}$ that never decelerate. Finally, these are the broadest FRs in our sample, with $F W H M_{s}=119.8^{\circ}$ and $F W H M_{s}=$ $118.4^{\circ}$, respectively. There is some suggestion that a shock develops ahead of event \#19 in the HI1 field of view, particularly visible in HI1-A. If true, this may be an ideal case for studying shock development in the interplanetary medium, which happens slowly and far from the Sun for this event thanks to its relatively slow, gradual acceleration.

Events \#20-21: There is a clear SDO/AIA brightening and post eruption loops associated with this event in an active region (AR 11289) near disk center, but the C2.9 flare taking place at about this time may be due to simultaneous activity near the east limb. We call this a flare-associated CME, but do not give a GOES designation in Table 1 due to this ambiguity. This is not the end of the ambiguity for this event, unfortunately. There are two consecutive MCs associated with the Wind ICME (see Figure 1), and close inspection of the imaging leads us to conclude that there are in fact two distinct CMEs erupting simultaneously from the same active region, which are very hard to separate. Our reconstruction therefore includes two Earth-directed CMEs launched simultaneously, CME1 and CME2. CME1 is somewhat faster and overtakes the CME2 front in COR1. CME1 is directed $\lambda_{s}=23^{\circ}$ west of Earth, while CME2 is at $\lambda_{s}=-2^{\circ}$ and with a more north-south orientation. Perhaps CME2 is associated with a part of the eruption above the post-eruption loops, while CME1 is more associated with an EUV dimming region west of the active region. In $\mathrm{LASCO} / \mathrm{C} 2$, the combined CME is seen as a partial halo, with the part of the halo to the west and northwest expanding faster than the part due north, a further indication that this is actually two distinct eruptions (CME1 and CME2, respectively). From STEREO-B's perspective the two CMEs end up almost perfectly superimposed after COR1, but separation between the leading edges of the two CMEs is apparent for STEREO-A. Finally, there is a jet of material towards the west (CME3), which has been modeled in the reconstruction as a lobular front (not an FR), although it comes nowhere near Earth. The order of arrival at Earth is CME1 hitting first and CME2 following only a couple hours later. The predicted encounter times of both are easily long enough to encompass both MC periods, so it is dubious whether we can 
really connect the two CMEs with the two MCs, but for purposes of our study an association is required. Both the arrival time order and FR orientation are suggestive of CME1 and CME2 being associated with the first and second MCs, respectively, so that is the assumption made in Tables 1 and 2. In the Wind data, near the ICME start time, there is a very narrow high density peak, which also has magnetic field signatures. It is tempting to associate this very unusual in situ signature somehow with the complex nature of this event, with apparent overlapping and interacting CMEs.

Event \#22: Our analysis involves the reconstruction of four separate CMEs: CME1 beginning at 9:20 on 2011 October 1, CME2 at 10:00, CME3 at 20:40, and CME4 at 1:00 on October 2. Neither CME1 nor CME3 are Earth-directed, with the former being directed west of Earth and the latter directed well to the east, more towards STEREO-B. CME3 is by far the fastest and brightest of the CMEs, with a clear shock that is included in the reconstruction. This CME is already mostly out of the field of view at the time shown in Figure 6. Our reconstruction has both CME2 and CME4 hitting Earth. The faster CME4 overtakes CME2 late in the HI1 field of view and ultimately hits Earth first, at a time close to the ICME and MC start time (see Figure 1), so this is the CME that we associate with the MC. Figure 6 shows the situation at about the time that CME2 is hitting Earth. There is always going to be some ambiguity in interpretation when two CMEs overlap like this, though. Could CME4 have accelerated the CME2 FR, so that it is still CME2 that accounts for the MC? Did CME4 deflect CME2 slightly to the east, meaning CME2 misses Earth entirely, which our reconstruction cannot reproduce due to the simple radial, self-similar expansion assumption? We prefer this latter interpretation, but we note that the predicted CME2 arrival time corresponds to the time of a big density increase near the end of the MC period in Figure 1, so perhaps that is the CME2 signature.

Event \#23: This reconstruction involving three CMEs is described in detail by Wood et al. (2016b), so we do not discuss it in great detail here. Only the second CME to erupt hits Earth. This is the most geoeffective CME in our sample, as measured by $D_{s t}$ (see Table 2$)$, despite being of only modest speed $\left(V_{\max }=720.3 \mathrm{~km} \mathrm{~s}^{-1}\right)$, and not being associated with a flare.

Event \#24: As the only $Q_{s}=5$ event in our sample, we have already discussed this event in Section 3.2 (see Figure 3), and also above in the discussion of event \#19, which resembles this event in many ways. With regards to its in situ signature, this is an instructive event for illustrating the ambiguities there can be connecting imaging and in situ signatures of a CME. We see nothing in the images that we associate with a shock, so the leading edge that we follow in the images is interpreted as the leading edge of the FR. The predicted Earth-arrival time agrees very well with the ICME start time marked in Figure 1, but the Wind in situ data does show a probable shock at that time, with the MC actually starting about 13 hours later. Does this mean that what we were following in the images was a shock all along? Did that front suddenly become a shock at some point in its interplanetary journey without us noticing? Given that our reconstruction has Earth encountering the southern leg of the FR, does this mean that there is more of a shock on the lateral parts of the FR than near the leading edge? There is not a very big density increase at the start of the MC that would suggest that we would have been following that in the images. It is notable that the predicted velocity is much higher than the observed velocity for the period between ICME arrival and the MC start time. This is to be expected for a shock and shock sheath, as images will track the shock propagation speed, which will be faster than the speed of material moving through the shock, which is what the in situ data will be measuring. It is also instructive to note that within the MC region the predicted and observed velocity curves agree well, consistent with this being CME ejecta (i.e., the FR).

Event \#25: Our reconstruction has Earth encountering the north leg of a north-south oriented FR. This CME is only just barely trackable into the HI2 field of view. The Wind data do not show very significant density, velocity, or field increases for this MC.

Event \#26: Our reconstruction includes a shock, which is clearly visible ahead of this bright, fast CME $\left(V_{\max }=\right.$ $1103.5 \mathrm{~km} \mathrm{~s}^{-1}$ ). This is easily the fattest FR in our entire sample, with $\Lambda_{s}=0.43$. This is a case where the lack of a clear trailing edge to the FR prevents this from being a very convincing case for an FR morphology, hence $Q_{s}=2$ in Table 2. A spheroidal blob model might have worked just as well. Our Earth-arrival time prediction is nicely consistent with the MC start time, but the Wind data show a much earlier arrival for the shock than our reconstruction predicts. 
Events \#27-28: There are many CMEs occurring in this time period, greatly complicating interpretation of the images. For our reconstruction, we decide in the interests of time to focus only on the two CMEs that we ultimately decide are Earth-directed: CME1 starting at 20:45 on 2012 July 3 and CME2 starting at 16:45 on July 4. These events are not very apparent from the frontal perspective of SOHO/LASCO. The brightest, fastest fronts seen passing over Earth in HI2-A on July 6 and 7 are other CMEs, not CME1 and CME2, only hints of which can be seen moving more slowly in the background. The two CMEs of interest are invisible in HI2-B. The difficulty seeing CME1 and CME2 in HI2 is indicative of the worsening viewing geometry for Earth-directed CMEs in mid-2012, which is why we did not continue to analyze events after 2012 July. Figure 6 shows the CME positions as CME1 is reaching Earth. We tentatively associate CME1 and CME2 with the first and second MCs identified in the Wind data, respectively (see Figure 1). The CME1 shock arrives at Earth in good agreement with the ICME start time, and the CME1 FR arrival agrees well with the start of the first MC. The arrival time of CME2 corresponds reasonably well with the beginning of the second MC.

\section{REFERENCES}

Al-Haddad, N., Nieves-Chinchilla, T., Savani, N. P., et al. 2013, Sol. Phys., 284, 129

Bevington, P. R., \& Robinson, D. K. 1992, Data Reduction and Error Analysis for the Physical Sciences (New York: McGraw-Hill)

Billings, D. E. 1966. A Guide to the Solar Corona (New York: Academic Press)

Bothmer, V., \& Schwenn, R. 1998, Ann. Geophys., 16, 1

Brueckner, G. E, et al. 1995, Sol. Phys., 162, 357

Burlaga, L. F. 1988, JGR, 93, 7217

Burlaga, L., Sittler, E., Mariani, F., \& Schwenn, R. 1981, JGR, 86,6673

Cane, H. V., \& Richardson, I. G. 2003, JGR, 108, 1156

Chen, J., Howard, R. A., Brueckner, G. E., et al. 1997, ApJ, 490, L191

Davies, J. A., Perry, C. H., Trines, R. M. G. M., et al. 2013, ApJ, 777, 167

Davis, C. J., Davies, J. A., Lockwood, M., et al. 2009, GRL, 36, L08102

DeForest, C. E., Howard, T. A., \& Tappin, S. J. 2011, ApJ, 738, 103

Démoulin, P., \& Dasso, S. 2009, A\&A, 498, 551

Démoulin, P. Nakwacki, M. S., Dasso, S., \& Mandrini, C. H. 2008, Sol. Phys., 250, 347

Eyles, C. J., Harrison, R. A., Davis, C. J., et al. 2009, Sol. Phys., 254,387

Farrugia, C. J., Osherovich, V. A., \& Burlaga, L. F. 1995, JGR, 100,12293

Gibson, S. E., \& Low, B. C. 1998, ApJ, 493, 460

Gopalswamy, N., Lara, A., Lepping, R. P., et al. 2000, GRL, 27, 145

Gopalswamy, N., Lara, A., Yashiro, S., et al. 2001, 106, 29207

Gopalswamy, N., Mäkelä, P., Xie, H., Akiyama, S., \& Yashiro, S. 2009, JGR, 114, A00A22

Gosling, J. T. 1993, JGR, 98, 18937

Gosling, J. T., Baker, D. N., Bame, S. J., et al. 1987, JGR, 92, 8519

Gosling, J. T., Hildner, E., MacQueen, R. M., et al. 1974, JGR, 79,4581

Gulisano, A. M., Démoulin, P., Dasso, S., \& Rodriguez, L. 2012, A\&A, 543, A107

Gulisano, A. M., Démoulin, P., Dasso, S., Ruiz, M. E., \& Marsch, E. 2010, A\&A, 509, A39

Harrison, R. A., Davies, J. A., Möstl, C., et al. 2012, ApJ, 750, 45

Hidalgo, M. A., \& Nieves-Chinchilla, T. 2012, ApJ, 748, 109

Hidalgo, M. A., Nieves-Chinchilla, T., \& Cid, C. 2002, GRL, 29, 1637
Howard, R. A, Moses, J. D., Vourlidas, A., et al. 2008, Space Sci. Rev., 136, 67

Howard, R. A., Sheeley, N. R., Jr., Michels, D. J., \& Koomen, M. J. 1985, ApJ, 90, 8173

Hu, Q., Qiu, J., \& Zheng, J. 2014, in Outstanding Problems in Heliophysics: From Coronal Heating to the Edge of the Heliosphere, ed. Q. Hu \& G. P. Zank (ASP Conf. Ser., Vol. 484), 78

Hundhausen, A. J., Sawyer, C. B., House, L., Illing, R. M. E., \& Wagner, W. J. 1984, JGR, 89, 2639

Janvier, M., Démoulin, P., \& Dasso, S. 2013, A\&A, 556, A50

Janvier, M., Démoulin, P., \& Dasso, S. 2014, A\&A, 565, A99

Janvier, M., Dasso, S., Démoulin, P., Masías-Meza, J. J., \& Lugaz, N. 2015, JGR, 120, 3328

Jian, L., Russell, C. T., Luhmann, J. G., \& Skoug, R. M. 2006, Sol. Phys., 239, 393

Kahler, S. 1987, Rev. Geophys., 23, 663

Kahler, S. W., \& Webb, D. F. 2007, JGR, 112, A09103

Kay, C., Opher, M., \& Evans, R. M. 2013, ApJ, 775, 5

Kay, C., Opher, M., \& Evans, R. M. 2015, ApJ, 805, 168

Kilpua, E. K. J., Mierla, M., Rodriguez, L., et al. 2012, Sol. Phys., 279, 477

Kilpua, E. K. J., Mierla, M., Zhukov, A. N., et al. 2014, Sol. Phys., 289, 3773

Krall, J. 2007, ApJ, 657, 559

Lepping, R. P., Acuña, M. H., Burlaga, L. F., et al. 1995, Space Sci.Rev., 71, 207

Lepping, R. P., Jones, J. A., \& Burlaga, L. F. 1990, JGR, 95, 11957

Lepping, R. P., Wu, C. -C., \& Berdichevsky, D. B. 2005, Ann. Geophys., 23, 2687

Lepping, R. P., Wu, C. -C., Berdichevsky, D. B., \& Szabo, A. 2011, Sol. Phys., 274, 345

Lepping, R. P., Wu, C. -C., Berdichevsky, D. B., \& Szabo, A. 2015, Sol. Phys., 290, 2265

Lindsay, G. M., Luhmann, J. G., Russell, C. T., \& Gosling, J. T. 1999, JGR, 104, 12515

Liu, Y. D., Luhmann, J. G., Möstl, C., et al. 2012, ApJ, 746, L15

Liu, Y., Thernisien, A., Luhmann, J. G., et al. 2010, ApJ, 722, 1762

Lugaz, N., Farrugia, C. J., Davies, J. A., et al. 2012a, ApJ, 759, 68

Lugaz, N. Kintner, P., Möstl, C., et al. 2012b, Sol. Phys., 279, 497

Lugaz, N., Vourlidas, A., \& Roussev, I. I. 2009, Ann. Geophys., 27, 3479 
Manchester, W. B., Gombosi, T. I., Roussev, I., et al. 2004a, JGR, 109, A01102

Manchester, W. B., Gombosi, T. I., Roussev, I., et al. 2004b, JGR, 109, A02107

Maričić, D., Vršnak, B., Dumbović, M., et al. 2014, Sol. Phys., 289,351

Marubashi, K. 1986, Adv. Space Res., 6, 335

Marubashi, K., \& Lepping, R. P. 2007, Ann. Geophys., 25, 2543

Mays, M. L., Taktakishvili, A., Pulkinnen, A., et al. 2015, Sol. Phys., 290, 1775

Millward, G., Biesecker, D., Pizzo, V., \& de Koning, C. A. 2013, Space Weather, 11, 57

Mishra, W., \& Srivastava, N. 2014, ApJ, 794, 64

Möstl, C., Amla, K., Hall, J. R., et al. 2014, ApJ, 787, 119

Möstl, C., Farrugia, C. J., Kilpua, E. K. J., et al. 2012, ApJ, 758, 10

Möstl, C., Farrugia, C. J., Temmer, M., et al. 2009, ApJ, 705, L180

Möstl, C., Temmer, M., Rollett, T., et al. 2010, GRL, 37, L24103

Nieves-Chinchilla, T., Colaninno, R., Vourlidas, A., et al. 2012, JGR, 117, A06106

Nieves-Chinchilla, T., Linton, M. G., Hidalgo, M. A., et al. 2016, ApJ, 823, 27

Ogilvie, K. W., Chornay, D. J., Fritzenreiter, R. J., et al. 1995, Space Sci.Rev., 71, 55

Owens, M. J., Merkin, V. G., \& Riley, P. 2006, JGR, 111, A03104

Richardson, I. G., \& Cane, H. V. 1996, JGR, 101, 27521

Riley, P., \& Crooker, N. U. 2004, ApJ, 600, 1035

Rollett, T., Möstl, C., Isavnin, A., et al. 2016, ApJ, 824, 131

Rouillard, A. P., Odstrcil, D., Sheeley, N. R., et al. 2011, ApJ, 735, 7

Russell, C. T., \& Shinde, A. A. 2005, Sol. Phys., 229, 323

Rust, D. M., \& Kumar, A. 1994, Sol. Phys., 155, 69

Savani, N. P., Owens, M. J., Rouillard, A. P., Forsyth, R. J., \& Davies, J. A. 2010, ApJ, 714, L128

Savani, N. P., Owens, M. J., Rouillard, A. P., et al. 2011, ApJ, 731,109

Schrijver, C. J., Aulanier, G., Title, A. M., Pariat, E., \& Delannée, C. 2011, ApJ, 738, 167

Schrijver, C., \& Title, A. M. 2011, JGR, 116, A04108

Sheeley, N. R., Jr., et al. 2008, ApJ, 675, 853

Stone, E. C., Frandsen, A. M., Mewaldt, R. A., et al. 1998, Space Sci.Rev., 86, 1
Temmer, M., Veronig, A. M., Peinhart, V., \& Vršnak, B. 2014, ApJ, 785, 85

Temmer, M., Vršnak, B., Rollett, T., et al. 2012, ApJ, 749, 57

Thernisien, A. F. R., Howard, R. A., \& Vourlidas, A. 2006, ApJ, 652,763

Thernisien, A., Vourlidas, A., \& Howard, R. A. 2009, Sol. Phys., 256, 111

Tousey, R. 1973, Space Research XIII, ed. M. J. Rycroft \& S. K. Runcorn (Berlin: Akademie-Verlay), 713

Vandas, M., \& Romashets, E. P. 2015, A\&A, 580, A123

Vandas, M., \& Romashets, E. P. 2003, A\&A, 398, 801

Vourlidas, A., Colaninno, R., Nieves-Chinchilla, T., \& Stenborg, G. 2011, ApJ, 733, L23

Vourlidas, A., Lynch, B. J., Howard, R. A., \& Li, Y. 2013, Sol. Phys., 284, 179

Vršnak, B., Temmer, M., Žic, T., et al. 2014, ApJS, 213, 21

Webb, D. F., Möstl, C., Jackson, B. V., et al. 2013, Sol. Phys., 285,317

Wood, B. E., \& Howard, R. A. 2009, ApJ, 702, 901

Wood, B. E., Howard, R. A., \& Linton, M. G. 2016a, ApJ, 816, 67

Wood, B. E., Howard, R. A., \& Socker, D. G. 2010a, ApJ, 715, 1524

Wood, B. E., Howard, R. A., Thernisien, A., Plunkett, S. P., \& Socker, D. G. 2009, Sol. Phys., 259, 163

Wood, B. E., Howard, R. A., Thernisien, A., \& Socker, D. G. 2010b, ApJ, 708, L89

Wood, B. E., Karovska, M., Chen, J., et al. 1999, ApJ, 512, 484

Wood, B. E., Lean, J. L., McDonald, S. E., \& Wang, Y. -M. 2016b, JGR, 121, 4938

Wood, B. E., Rouillard, A. P., Möstl, C., et al. 2012a, Sol. Phys., 281,369

Wood, B. E., Wu, C. -C., Howard, R. A., Socker, D. G., \& Rouillard, A. P. 2011, ApJ, 729, 70

Wood, B. E., Wu, C. -C., Rouillard, A. P., Howard, R. A., \& Socker, D. G. 2012b, ApJ, 755, 43

Wu, C. -C., Dryer, M., Wu, S. T., et al. 2011, JGR, 116, A12103

Wu, C. -C., \& Lepping, R. P. 2015, Sol. Phys., 290, 1243

Wu, C. -C., \& Lepping, R. P. 2016, Sol. Phys., 291, 265

Wu, S. T., Andrews, M. D., \& Plunkett, S. P. 2001, Space Sci. Rev., 95, 191

Zhao, X. P., Plunkett, S. P., \& Liu, W. 2002, JGR, 107 (A8), SSH 13-1 